\title{
DEMOCRAZIA E FEDERALISMO. UN'ANALISI COMPARATA
}

\author{
di Alfred Stepan
}

\section{Democrazia, federalismo e plurinazionalità}

In questo articolo proporrò alcune riflessioni sul rapporto fra democratizzazione, federalismo e nazionalismo, prendendo spunto da lavori già avviati su questa tematica insieme a Juan Linz (Linz 1997).

La prima fondamentale distinzione da cui occorre partire è quella tra un sistema democratico federale e un sistema non democratico che presenta caratteristiche federali. In senso stretto, solo una democrazia costituzionale è in grado di fornire delle $\mathrm{ga}$ ranzie credibili e dei meccanismi istituzionali che assicurino il rispetto delle prerogative legislative delle unità sub-nazionali. In una democrazia le specificità della formula federale, che sono state costituzionalmente adottate, struttureranno quotidianamente molti dei processi politici, come le procedure di approvazione delle leggi più importanti a livello centrale o ai livelli subnazionali e la definizione e il rispetto dei confini giurisdizionali tra il centro e le sotto-unità. In un sistema non democratico, invece, non è detto che il federalismo possa strutturare in modo significativo questi processi politici ${ }^{1}$. Se si accetta, come faccia-

1 Questo non significa negare che in certe circostanze le caratteristiche federali di alcuni sistemi non democratici possano diventare estremamente importanti. Per esempio, durante il periodo stalinista in Urss era lo Stato-partito guidato da Stalin a decidere le politiche. Questo accadeva nonostante il grande numero di diritti di sovranità attribuiti dalla Costituzione alle Repubbliche sovietiche. Tuttavia, con l'indebolimento dello Stato-partito alla fine degli anni ' 80 e con l'approvazione di un decreto di competizione elettorale a livello repubblicano, le stesse caratteristiche federali del sistema sovietico, nel nuovo contesto politico, fornirono alle élites delle Repubbliche significative opportunità di «mobilitazione delle risorse» per ricostituire le loro basi di potere enfatizzando le richieste di sovranità (Linz e Stepan 1996, 366-400). Altri due casi sono significativi in proposito: il Brasile, in cui il federalismo ha mantenuto una qualche im-

RIVISTA ITALIANA DI SCIENZA POLITICA / a. XXVIII, n. 1, aprile 1998 
mo Linz ed io, la definizione di sistema federale proposta da R.A. Dahl, allora solo una democrazia può essere un sistema federale in senso proprio. Per federalismo, infatti, Dahl intende «un sistema in cui alcune materie cadono esclusivamente sotto la competenza di talune unità locali (cantoni, stati, province) e si trovano costituzionalmente oltre la portata dell'autorità del governo nazionale, e in cui certe altre materie si trovano costituzionalmente al di fuori della portata dell'autorità delle unità più piccole» (Dahl 1986, 114, corsivo nel testo). Solo una democrazia è in grado di costruire sistemi costituzionali, legislativi e giudiziari relativamente autonomi che corrispondono ai requisiti di federalismo appena indicati. Pertanto, la mia attenzione in questo articolo è sul federalismo nei sistemi democratici, con tutto quello che ne consegue.

Se si vogliono fare dei progressi a livello comparato, è necessario analizzare la differenza tra il funzionamento politico dei sistemi federali democratici e non democratici. Alcuni dei principali studi sul federalismo non fanno questa distinzione. Per esempio, W.H. Riker nel suo articolo del 1975 sul federalismo, molto spesso citato, afferma che Canada, Stati Uniti, Yugoslavia e Urss rispondono tutti ai suoi criteri di federalismo e classifica gli Stati Uniti e la Yugoslavia come appartenenti entrambi al sotto-tipo «federalismo centralizzato» (Riker 1975). Ricomprendere Yugoslavia e Stati Uniti nella stessa categoria analitica offusca le differenze più significative esistenti tra le strutture di potere e le dinamiche politiche dei due sistemi politici ${ }^{2}$.

Un'altra distinzione fondamentale è tra sistemi federali il cui scopo iniziale è quello di «diventare uniti» (come together) e sistemi federali il cui scopo è di «mantenersi uniti» (bold together). L'idea di una federazione «per diventare uniti» si basa

portanza politica anche durante le due fasi non democratiche del $1930-45$ e del 1964 85, e il Messico, in cui il federalismo sta assumendo un ruolo sempre più importante nell'agonia di uno dei sistemi partitici non democratici di più lunga durata del mondo.

2 Ad esempio, è proprio perché la Yugoslavia non era un sistema democratico che il suo «sistema quasi-federale» ha potuto funzionare in modo pacifico fino a quando lo Stato-partito ha avuto un ruolo cruciale nella guida del paese. Una volta che le elezioni hanno cominciato a infondere un po' di vita in quel sistema federale inerte e incompleto, il sistema ha cominciato a frammentarsi. Questo punto è di grande importanza per l'analisi comparata del federalismo. Se si esclude il caso tedesco di «una nazione in due stati», nel 1989 nell'Europa dell'Est vi erano otto stati non democratici, di cui cinque unitari e tre con alcune caratteristiche quasi-federali. I cinque stati unitari sono ancora tali nel 1997. I tre stati quasi-federali si sono trasformati in ventidue paesi indipendenti, molti dei quali etnocrazie maggioritarie non democratiche. 
sul modello americano. Con la Convenzione di Filadelfia, nel 1787, le unità precedentemente sovrane stipularono quello che Riker chiama un «contratto federale» per unirsi, ponendo la loro sovranità in comune in una nuova federazione che lasciò poteri residuali ai singoli stati. Per gli stati sovrani che decisero di unirsi un aspetto cruciale del contratto federale era che la nuova federazione veniva costruita con caratteristiche verticali e orizzontali in grado di vincolare il demos a livello centrale. Secondo Riker una federazione funzionante deve essere costruita sulla base di un contratto di questo tipo.

Alcune delle più importanti federazioni, tuttavia, sono la risultante di una logica storica e politica completamente diversa. L'India nel 1948, il Belgio nel 1969 e la Spagna nel 1975 erano tutti sistemi politici con forti tratti unitari. Nonostante ciò, $\mathrm{i}$ leader politici in tutte e tre queste società multiculturali decisero costituzionalmente che il modo migliore per «mantenersi uniti» in una democrazia era di delegare il potere e trasformare i loro sistemi politici a rischio in federazioni. Così la costituzione indiana del 1950, quella spagnola del 1978 e quella belga del 1993 sono tutte e tre federali.

Esaminiamo brevemente le caratteristiche del federalismo indiano, che è del tipo «non contrattuale» e orientato al «mantenersi uniti», per mettere a fuoco le differenze che lo separano dal federalismo di tipo «contrattuale» e orientato al «diventare uniti» proprio dell'esperienza nord-americana. Il Presidente della commissione incaricata di redigere la Costituzione indiana, B.R. Ambedkar, illustrando il progetto costituzionale ai membri dell'Assemblea costituente, sottolineò esplicitamente che il fine della Costituzione indiana era il mantenimento dell'unità del paese. Affermò inoltre che i principi e i meccanismi alla base della costituzione erano profondamente diversi da quelli su cui si fondava la Costituzione americana. Egli riteneva che l'India fosse già un sistema politico eterogeneo caratterizzato da una sostanziale unità, ma che per mantenere questa unità in condizioni democratiche fosse necessario dare vita ad una federazione. Ambedkar disse ai membri dell'Assemblea: «...L'uso del termine Unione è intenzionale... La Commissione redigente ha voluto rendere esplicito che se l'India dovesse diventare una federazione, tale federazione non sarebbe il risultato di un accordo tra gli stati per unirsi in una federazione e, di conseguenza, non essendo la federazione il risultato di un accordo, nessuno stato avrebbe il diritto di secedere da essa... Pertanto, anche se 
il paese e il popolo fossero divisi in stati differenti per ragioni di convenienza amministrativa, il paese rimarrebbe un'entità integrale, il popolo un solo popolo, che vive sotto un unico comando che deriva da un'unica fonte» ${ }^{3}$.

In un'accurata analisi delle intenzioni dei padri fondatori della Costituzione indiana, Mohit Bhattacharya sottolinea che quando Ambedkar presentò il testo alla Assemblea costituente nel novembre del 1948, tanto la separazione tra Pakistan e India quanto l'integrazione, benché ostacolata e occasionalmente perseguita con strumenti coercitivi, di tutti i 568 principati erano oramai un fatto compiuto. Quando Ambedkar presentò il testo della Costituzione ${ }^{4}$, pertanto, una situazione di patto tra unità sovrane, tipico del federalismo «per diventare uniti» di Riker, di fatto non esisteva. Secondo Bhattacharya la motivazione principale dei padri costituenti fu quella di mantenere unito il livello centrale di governo. "Quello che da ultimo emerse fu un "sistema federale decentratore" (devolutionary federation), dal momento che uno stato fondamentalmente unitario decise di trasferire, attraverso un lungo processo evolutivo, i poteri alle unità territoriali. (...) [Una volta] superato il problema dell'integrazione dei principati grazie alla separazione di India e Pakistan, la stessa "situazione federale" di fatto venne meno (...) come del resto anche la situazione di patto. (...) Gli artefici della costituzione rivelarono il loro pragmatismo. Riservarono grande attenzione all'autorità centrale che avrebbe dovuto mantenere unita la nazione» (Bhattacharya 1992, 101-102).

3 Il discorso di Ambedkar è interamente riportato in India Constituent Assembly (1951, 31-44). Corsivo aggiunto.

4 Se la separazione non fosse avvenuta e se i territori musulmani che ora corrispondono al Pakistan e al Bangladesh avessero inviato propri rappresentanti all'Assemblea costituente, sarebbe sicuramente prevalsa una situazione di trattativa/negoziazione. In tale contesto, anche il potere di contrattazione dei principati sarebbe aumentato (soprattutto quello dei musulmani che governavano sui principati dell'India centrale, come Hyderabad). Una volta che ebbe inizio la violenta separazione, tuttavia, sia i leader del Partito del Congresso nell'Assemblea costituente che l'ultimo Vice-Re inglese, Lord Mountbatten, si resero conto che di fatto esisteva già una nuova situazione geo-politica. In questo nuovo quadro, nel settembre 1948, il Governo Nehru ricorse alla forza militare per integrare il principato più esteso, Hyderabad. Mountbatten, nel corso di numerose occasioni sia formali che informali, fece sapere ai principati interni ai nuovi confini dello Stato indiano che non esisteva più una situazione di trattativa e anzi li sollecitò ad unirsi all'Unione Indiana perché, se non lo avessero fatto, non sarebbero comunque stati ammessi a far parte del Commonwealth britannico come stati indipendenti. I due testi principali sulla situazione di non-trattativa seguita alla separazione sono Menon (1956) e Copland (1997). 
Il terzo punto fondamentale per una nuova agenda di ricerca sul federalismo democratico dovrebbe riguardare il nazionalismo e, soprattutto, il federalismo nei sistemi politici multiculturali e plurinazionali. Sfortunatamente, come i comparativisti hanno da approfondire la relazione tra federalismo e democrazia, così noi dobbiamo approfondire in misura ancora maggiore la relazione tra nazionalismo (e plurinazionalità) e democrazia. Già dal 1986, tre anni prima della caduta del muro di Berlino, erano disponibili due importanti filoni di letteratura che avrebbero dovuto aiutarci a riflettere sulla complessa relazione esistente tra democratizzazione e (pluri)nazionalità. Nel 1983 Ernest Gellner pubblicò il suo magistrale Nations and Nationali$\mathrm{sm}$, lo stesso anno in cui uscì un altro classico moderno sul nazionalismo, Imagined Communities: Reflections on the Origin and Spread of Nationalism di Benedict Anderson. Nel 1986 venne pubblicato, a cura di O'Donnell, Schmitter e Whitehead, l'opera in quattro volumi Transitions from Authoritarian Rule, che immediatamente contribuì a creare il settore della «transizionologia» (transitology).

Quello che mi colpisce è che due filoni di letteratura che, possiamo dire retrospettivamente, avrebbero avuto molto da apprendere l'uno dall'altro, siano rimasti in realtà separati e non comunicanti. Nei quattro volumi sulle transizioni democratiche, il nazionalismo non viene mai tematizzato come una questione rilevante e nemmeno gli viene dedicato un capitolo specifico. La parola nazionalismo appare soltanto nell'indice analitico di uno dei quattro volumi, quello sul Sud Europa, e i riferimenti si limitano ad una pagina per la Spagna, una per il Portogallo e due per la Grecia. In nessuno dei quattro volumi appaiono i nomi di Gellner e di Anderson. In quanto autore di uno degli articoli comparati pubblicati in questa serie di volumi anch'io sono in parte responsabile di questa omissione. Dal canto loro, né Gellner né Anderson affrontano nei rispettivi volumi il tema della democrazia ed infatti questa parola non compare nell'indice analitico dei loro libri.

La mia opinione è che uno dei problemi più urgenti che tanto i teorici della democrazia quanto i professionisti della politica si trovano ad affrontare è come riconciliare nazionalismo e democrazia, soprattutto in contesti multi-etnici. Il federalismo

5 I quattro volumi, pubblicati separatamente, trattano rispettivamente di temi di teoria generale, del Sud Europa, dell'America Latina e delle prospettive comparate. 
(insieme con alcune pratiche consociative) spesso rappresenta una delle formule politiche potenzialmente attraenti (ma anche potenzialmente pericolose) che coloro che intendono creare un sistema democratico in società plurinazionali devono prendere in considerazione. Per questo i due dibattiti sulla democratizzazione e sul nazionalismo che fino ad ora hanno proceduto su binari separati, dovrebbero invece dar vita ad un dialogo costante soprattutto se si considera seriamente la possibilità del federalismo democratico plurinazionale (Linz e Stepan 1996; Linz 1997; Stepan 1997). Ora che abbiamo dimostrato come, ai fini dell'analisi comparata, questi tre elementi chiave - democrazia, federalismo e nazionalità - siano necessariamente interrelati, vorrei analizzare sinteticamente due dei molti aspetti del federalismo democratico che possono essere affrontati con più efficacia connettendo $i$ tre concetti in un unico schema di analisi.

\section{Il federalismo democratico: identità duali ma complementari}

Se vogliamo che il concetto di federalismo rappresenti un utile strumento nell'analisi comparata dei processi di democratizzazione, è necessario sviluppare le implicazioni per le identità e le lealtà politiche dei requisiti minimi del federalismo democratico proposti da Dahl. I sistemi politici democratici probabilmente dovrebbero essere definiti federali solo se soddisfano due criteri. Innanzitutto, all'interno di uno stato devono esistere delle sotto-unità politico-territoriali definite, il cui elettorato sia composto esclusivamente da cittadini appartenenti all'unità locale stessa $e$ in cui esistano degli ambiti di autonomia e sovranità giurisdizionale e legislativa garantiti costituzionalmente. In secondo luogo, deve esistere una unità politica statale caratterizzata da un organo legislativo eletto dall'intera popolazione dello stato federale e che dispone di ambiti decisionali e legislativi garantiti costituzionalmente.

Se si accetta questa definizione ne consegue che in un sistema democratico federale consolidato, più i cittadini si sentono leali verso entrambi i livelli di sovranità democraticamente legittimati - ognuno con un proprio ambito di azione garantito costituzionalmente - più la federazione è salda dal punto di vista democratico. Idealmente, quindi, i cittadini di un sistema democratico federale dovrebbero avere identità politiche duali ma com- 
plementari. Se infatti i cittadini di una sotto-unità territoriale e i loro leader eletti a livello sub-nazionale percepissero che il governo centrale non fornisce loro i beni, la sicurezza o l'identità che essi considerano importanti e che da sola l'unità locale non è in grado di garantire, allora la loro fedeltà verso il livello di governo centrale si indebolirebbe. Potenzialmente ciò potrebbe contribuire a creare una constituency favorevole ad una politica secessionista, fino a generare le basi di una politica di alienazione o un senso di sfruttamento, che certo non favorirebbero il consolidamento democratico. Allo stesso modo, se molti cittadini dello stato federale e i loro leader nazionali percepissero che il sistema federale assicura benefici scarsi ma impone costi politici ed economici elevati $e$ che i costi di exit sono relativamente bassi, la stabilità del sistema federale democratico sarebbe ugualmente in pericolo.

Il federalismo è dunque una formula potenzialmente attraente per alcuni tipi di società plurinazionale, ma la politica di formazione e mantenimento di identità duali e complementari necessita di maggiori riflessioni e ricerche. I sondaggi di opinione sono uno strumento particolarmente promettente per esplorare le circostanze politiche in cui la lealtà verso la «nazione-stato» al livello centrale può aumentare mentre cresce anche la lealtà verso sotto-unità non secessioniste, ma comunque nazionaliste.

\section{Un federalismo che limiti la maggioranza}

La distinzione tra federalismo «per unirsi» e federalismo «per mantenersi uniti» apre la strada al riesame di una delle principali questioni normative. Un implicito, e spesso esplicito, argomento normativo a favore del federalismo è che esso protegge i diritti individuali contro un livello centrale troppo potente o persino contro la «tirannia della maggioranza». William $\mathrm{H}$. Riker ha esplicitamente affermato che uno degli aspetti che più attrae del federalismo è il fatto che esso, contribuendo a creare un governo limitato, esercita un controllo sulle maggioranze populiste. «L'ideale populista richiede che i governanti trasformino le decisioni popolari in legge, in tempi rapidi e certi, sulla base di una piattaforma elettorale» (Riker 1982, 247). Dal punto di vista normativo, Riker è a favore di un legislativo multi-camerale proprio perché ritiene che possa contribuire a limitare le maggioranze populiste. Egli richiama l'attenzione sul fatto che 
negli Stati Uniti il potere legislativo multi-camerale «è in realtà composto da tre camere: il Presidente, il Senato e la Camera dei rappresentanti, ognuna basata su differenti divisioni dei cittadini in constituencies. Le diverse constituencies hanno mantenuto separati gli interessi di coloro che governano» (Riker 1982, 250). Per Riker, il secondo significativo strumento del federalismo americano per limitare il governo è rappresentato dalla divisione dell'autorità tra governi nazionali e locali e soprattutto dal suo effetto di frammentazione sui partiti. A questo proposito egli scrive: «Questo è il famoso federalismo americano, copiato in mezzo mondo. Il freno costituzionale non è comunque rappresentato dalla divisione legale dei doveri tra governo centrale e governi locali, ma piuttosto dalla conseguente localizzazione dei partiti politici che rende impossibile una loro leadership nazionale» (Riker 1982, 250). Per Riker i vincoli positivi che il federalismo americano promuove hanno a che fare con il fatto che «il multi-cameralismo e il federalismo hanno rafforzato il localismo nei partiti e questo, a sua volta, ha spinto i governanti ad usare strumenti di persuasione piuttosto che strumenti di controllo. L'effetto globale che ne deriva è che nessuna politica è modificata in modo tanto rapido e tanto radicale da produrre conseguenze molto negative su qualcuno» (Riker 1982, 253).

Tuttavia, possiamo davvero affermare che nei sistemi federali plurinazionali, soprattutto nelle fragili democrazie di recente formazione, sia sempre normativamente desiderabile o politicamente utile avere una «localizzazione dei partiti politici» che renda impossibile una leadership nazionale? Ciò non è per niente chiaro. Prendiamo ad esempio il ruolo dei partiti nazionali alle prime elezioni politiche in stati federali plurinazionali. Nelle prime elezioni in Nigeria, tenutesi nel 1959, lo stato era diviso in tre regioni (settentrionale, occidentale e orientale), in ognuna delle quali vinse un partito regionale a base etnica ottenendo il controllo politico della regione. Nel sistema legislativo federale di fatto non venne eletto alcun rappresentante di un partito nazionale. Questo risultato contribuì direttamente a far emergere dalle composite fratture del regionalismo etnico-territoriale un intenso conflitto politico e portò infine, in seguito al tentativo secessionista del Biafra, allo scoppio della guerra civile. In Yugoslavia le prime elezioni competitive si tennero a livello delle repubbliche e vennero tutte vinte da partiti nazionalisti a base regionale ed etnica. La guerra civile in Yugoslavia scoppiò prima che si svolgessero elezioni nazionali. Al contrario, in Spagna 
si tennero contemporaneamente le elezioni nazionali e regionali. I partiti nazionali diedero vita al governo centrale $e$ a dodici dei quattordici governi locali. In India, dopo le elezioni tenutesi contemporaneamente a livello nazionale e sub-nazionale, il partito nazionale del Congresso formò il governo centrale $e$ tutti i governi statali. Chiaramente, se l'obiettivo è il consolidamento democratico in stati multiculturali e plurinazionali, si può affermare che l'esistenza di partiti nazionali è utile.

Il ruolo dei partiti nazionali sembra essere particolarmente importante soprattutto se il fine principale è quello di «mantenere unito» il sistema federale in una forma che riconcili le diversità culturali con l'efficacia del processo decisionale. Tale riconciliazione era proprio ciò che l'Assemblea costituente indiana intendeva perseguire. Per citare di nuovo Ambedkar «(...) la Costituzione ha cercato di creare strumenti e metodi grazie ai quali l'India potesse essere una federazione di stati e allo stesso tempo avere uniformità in tutte le questioni essenziali per mantenere l'unità del paese. I mezzi adottati dalla Costituzione sono tre: un unico sistema giudiziario; uniformità nelle leggi fondamentali, sia civili che penali; un apparato burocratico, che per quello che riguarda i posti importanti, sia comune a tutto il paese». Ambedkar continua sottolineando la distinzione basilare tra il modello americano di divisione dei poteri, che permette un governo diviso a livello centrale, e quello indiano di fusione dei poteri che richiede a livello centrale almeno la maggioranza di una coalizione di partiti. «La forma di governo americana è presidenziale (...) Al contrario, la Costituzione [indiana] ha introdotto un sistema parlamentare. I due sistemi sono profondamente differenti. Il sistema esecutivo americano non è parlamentare, ossia non dipende dall'esistenza di una maggioranza al Congresso (...) Un esecutivo di tipo parlamentare dipende invece dalla maggioranza in Parlamento e deve dimettersi nel momento in cui viene meno la fiducia della maggioranza dei membri del Parlamento» (India Costituent Assembly 1951, 124).

Passiamo ora ad affrontare alcune questioni critiche, sia di natura normativa che concettuale, riguardo alla democrazia e ad esplicitare ciò che intendo quando uso le espressioni «demo-vincolante» e «demo-emancipante» ${ }^{6}$. Se si ritiene il consolidamento

6 Nella prima versione di questo articolo ho fatto uso dell'espressione «maggioranza vincolata» invece che «demo-vincolato». Scott Mainwaring e Gerry Mackie hanno 
democratico un obiettivo normativo desiderabile e se si riconosce che nella realtà tale consolidamento spesso non è raggiunto, allora è utile riflettere su quale insieme di valori, in senso probabilistico, possa essere associato alla promozione o all'inibizione del consolidamento democratico. Io credo che si favorisca il consolidamento democratico se sono garantiti tre valori importanti come la libertà, l'uguaglianza e l'efficacia in modo tale che nessuno di essi sia considerato secondario e che tutti si rinforzino a vicenda.

La libertà dei cittadini individuali è ovviamente un valore positivo. In una democrazia, essa implica che la maggioranza, comunque costituita, non debba imporre alle minoranze politiche che violino i diritti individuali. Il costituzionalismo e il governo della legge sono le principali istituzioni democratiche che aiutano a preservare la libertà e i diritti individuali. Molti pensatori liberali, soprattutto di tradizione americana, ritengono che il federalismo abbia un ruolo decisivo, per alcuni il ruolo decisivo, nel limitare la «tirannia della maggioranza». Un altro valore nelle democrazie moderne è l'uguaglianza dei cittadini nel sistema politico, ossia l'uguaglianza dei membri del demos. In senso stretto questo significa «un cittadino, un voto». In senso più generale uguaglianza può anche significare che il demos si assume l'impegno di portare tutti i cittadini alla condivisione di un insieme di diritti di base e di benessere. Da ultimo, affinché la democrazia possa perpetuarsi e affinché le nuove democrazie si possano consolidare, le istituzioni di governo dovrebbero essere costituite in modo tale da permettere al demos nazionale e ai demoi delle sotto-unità di essere governati con un grado accettabile di efficacia. L'efficacia, diversamente dalla libertà, non è un valore collegato unicamente alla democrazia. Anche regimi non democratici possono aspirare all'efficacia. Ma se consideriamo la democrazia come un insieme di istituzioni vive, di valore e storicamente precarie, vi sono ragioni per credere che uno stato democratico con un basso grado di efficacia abbia minori possibilità di durare di

sollevato una serie di questioni concettuali riguardo al primo termine, di cui li ringrazio. L'espressione «demo-vincolante» non implica che esista sempre una maggioranza o che abbia sempre ragione. Io credo che in alcuni sistemi federali, soprattutto in quelli multiculturali e plurinazionali, vi possa essere spazio per pratiche consociative non maggioritarie sulle quali vi sia consenso. Demos, tuttavia, implica l'esistenza di una categoria che include «tutti i cittadini dello stato». Uno degli obiettivi principali di questo articolo è proprio quello di dimostrare come tipi differenti di sistemi federali democratici «vincolino il demos» mentre alcuni sono in grado di «emanciparlo». 
una democrazia con un grado medio o alto di efficacia. Dove è possibile si dovrebbe evitare di creare istituzioni di governo per il demos e per i demoi sub-nazionali che rendano particolarmente difficile perseguire l'efficacia.

Se si accetta che «libertà», «uguaglianza» ed «efficacia» sono tutte importanti per una democrazia di qualità, allora bisogna chiedersi come questi tre principi siano tra loro collegati in un sistema federale democratico. Una forte corrente del pensiero liberale americano sottolinea il contributo del federalismo alla libertà. Riker, come abbiamo visto, è un esponente di questa tradizione. Alcuni autori particolarmente interessati a prevenire la «tirannia della maggioranza» prendono in considerazione non solo l'idea di «super-maggioranze» ma persino l'unanimità legislativa in sistemi legislativi multipli' ${ }^{7}$. Questo ovviamente precluderebbe la tirannia della maggioranza.

$\grave{E}$ di grande vantaggio se per alcune questioni costituzionali fondamentali e controverse si raggiungono accordi consensuali, quasi unanimi. Tuttavia, sia teoricamente che empiricamente, una «regola di unanimità» o persino la richiesta di una rigida super-maggioranza per le decisioni di governo ordinarie è in forte contrasto con i valori di efficacia ed eguaglianza. La richiesta di una super-maggioranza rende la legislazione più difficile da approvare e dà alle minoranze un grande potere di veto $^{8}$. Infatti, nel caso di una forma di federalismo demo-vincolante estrema, alcuni legislatori, pur rappresentando probabilmente meno del $10 \%$ dell'elettorato, potrebbero costituire un gruppo vincente paralizzante (blocking win-set). In simili circostanze questi decisori, anche se fossero programmaticamente favorevoli alla legislazione, potrebbero essere strutturalmente tentati di sfruttare la loro posizione per ottenere rendite di posizione (rent-seeking rewards). Chiaramente, piccoli blocking win-sets costituzionalmente radicati nel sistema federale democratico sollevano legittime domande rispetto al principio «un cittadino un voto».

7 Per un'utile discussione di questa dimensione normativa del pensiero politico americano si veda Goodin (1996).

$8 \mathrm{Se}$ in un sistema federale si dovesse richiedere una super-maggioranza per tutte le decisioni, allora alcune «condizioni di partenza» potrebbero essere molto meglio di altre. Ad esempio, se le «condizioni di partenza» comprendono una grande diseguaglianza (come era la schiavitù nel Sud degli Stati Uniti) questo accrescerebbe i problemi potenziali più che in un sistema federale caratterizzato da diffusa eguaglianza sociale ed economica. 
Pertanto, dal punto di vista politico, blocking win-sets costituzionalmente radicati possono potenzialmente sollevare problemi per l'efficacia e la legittimità del funzionamento democratico. Per esempio, in un paese potrebbe verificarsi che per un periodo prolungato una solida maggioranza di leader politici e una grande maggioranza dell'elettorato ritengano necessari importanti cambiamenti. Tuttavia, se la Costituzione federale prevede meccanismi per facilitare «gruppi vincenti» di veto ad opera di piccole minoranze, l'efficacia e la legittimità di quel sistema democratico potrebbero essere erosi dalle caratteristiche federali demo-vincolanti. Se molti cittadini sono convinti che un governo popolare che persegue politiche popolari viene paralizzato dalle stesse istituzioni democratiche (caso estremo di sistemi federali demo-vincolanti) è possibile che si crei un contesto in cui la maggior parte della legislazione di fatto non viene bloccata ma è approvata attraverso decreti governativi e con un ampio sostegno popolare. In termini di efficacia questa situazione può rivelarsi sia positiva che negativa. Di certo non facilita il consolidamento democratico, che è invece favorito se l'approvazione delle leggi avviene all'interno del processo legislativo democratico e non all'esterno'.

9 Due possibili argomentazioni contro la tesi che intendo sostenere in questo articolo - che il Brasile è un caso estremo sul continuum «demo-vincolante» - sono che il Presidente Fernando H. Cardoso, democratico riformista in carica dal 1995, raramente è stato bloccato nel processo di implementazione delle nuove misure di riforma perché $a$ ) ha fatto uso di decreti presidenziali e $b$ ) la maggior parte della legislazione da lui formalmente presentata non sotto forma di decreto al Congresso è stata approvata (Figueiredo e Limongi 1997). Pur non contestando i dati contenuti nel dettagliato e accurato lavoro appena citato, vorrei fare due osservazioni. Primo, il fatto che il Presidente Cardoso, che aspira a consolidare le istituzioni democratiche del Brasile, debba approvare attraverso decreti così tante leggi che godono del sostegno popolare e che sono cruciali per l'efficacia del governo, è un modo improprio per migliorare i valori democratici di libertà, uguaglianza ed efficacia. Sotto la guida di un presidente meno capace e meno impegnato sul fronte democratico, l'elemento demo-vincolante del federalismo brasiliano contribuirebbe alla formazione di quella che O'Donnell definisce una «democrazia delegativa». La seconda osservazione è di natura metodologica (ed estremamente politica). Solo i leader politici hanno così tanto capitale e risorse politici. E sanno anche come farli pesare. Se un forte gruppo vincente di minoranza si oppone a molte delle loro proposte di policy, allora essi sapranno essere parsimoniosi nelle misure da approvare con l'appoggio di tale minoranza. Alla luce di questa prospettiva metodologica che cosa è più importante? Il fatto che la maggior parte delle misure che il Presidente propone al Congresso vengono approvate oppure che, a causa di ciò che egli considera un gruppo vincente paralizzante, il Presidente decide di non proporre formalmente al Congresso la maggior parte delle misure che vorrebbe approvare? Sulla base delle interviste che ho fatto ai ministri all'inizio del governo Cardoso e due anni dopo, ritengo che la seconda sequenza sia quella politicamente più significativa. 
Prima di proseguire, indico brevemente perché lo studio del federalismo è così importante per l'analisi della democrazia moderna. Se l'unità d'analisi prescelta è costituita dai singoli paesi, allora non più del $10 \%$ degli stati appartenenti alle Nazioni Unite è federale. Se, invece, l'unità d'analisi è rappresentata da tutti gli individui che vivono in sistemi democratici consolidati, si può dire che la maggioranza della popolazione vive in sistemi federali. Questo dato è certamente correlato alle dimensioni dei sistemi democratici federali. La democrazia più popolata del Nord America, gli Stati Uniti, è federale. Lo stesso si può dire per la Germania in Europa occidentale e per l'India in Asia. Inoltre molte altre democrazie di lunga data come il Canada, l'Australia, la Svizzera e l'Austria sono federali. Infine, due sistemi democratici europei hanno recentemente optato per forme federali (Elazar 1994). Nel 1979 in Spagna furono approvati gli Statuti di autonomia che hanno trasformato il paese in un sistema federale di tipo asimmetrico (Linz 1989; Agronoff 1994). Il Belgio nel 1993, in seguito a una profonda revisione costituzionale, ha dato vita ad un sistema federale con numerosi arrangiamenti di tipo consociativo (Hooghe 1993; Senelle 1996; Swenden 1997). Se la questione analitica riguarda paesi che non sono democrazie di vecchia data, ma nei quali il problema della transizione democratica è ormai risolto, e se in essi la natura del federalismo è parte rilevante nel dibattito sulle prospettive democratiche, allora, per ragioni di analisi comparata, Brasile e Russia vanno aggiunti alla lista dei sistemi federali importanti (Ordershook 1996; Ordershook e Svetsova 1997; Solnick 1995). Il paese più popolato dell'Africa nera, la Nigeria, che ha conosciuto a fasi alterne il federalismo e la democrazia, potrebbe per certe fasi fare anch'esso parte di questa categoria (Suberu 1997). Si può anche pensare al dibattito sul federalismo e l'Unione europea - forma democratica di cui si sta tanto discutendo in questi anni, a cui alcune persone in Europa aspirano e che molte altre temono. Infine, se volessimo identificare alcuni paesi che non sono mai stati democratici ma che se lo diventassero probabilmente sceglierebbero un sistema federale, allora dovremmo aggiungere anche il Messico, l'Indonesia e la Cina alla lista dei paesi per i quali l'analisi del federalismo è centrale per lo studio dei possibili modelli di transizione democratica ${ }^{10}$.

10 Ad esempio Linz ed io abbiamo altrove sostenuto che l'Indonesia e la Cina rischierebbero una disgregazione violenta come quella dell'Urss e della Yugoslavia qualo- 


\section{Oltre l'approccio di Riker}

Fra i molti importanti studiosi del federalismo vi sono Kenneth C. Wheare, un autorevole costituzionalista, e Daniel J. Elazar, il fondatore di Publius, la rivista periodica dedicata al federalismo $^{11}$. Tuttavia, lo studioso che ha maggiormente influenzato la scienza politica sul federalismo è Riker e non si può andare troppo lontano senza ripartire dal suo pensiero, magari per proporre delle buone ragioni per raffinarlo o per rifiutarlo. Riker è stato uno dei fondatori intellettuali della teoria della scelta razionale in scienza politica, ha contribuito al dibattito sulla teoria delle coalizioni soprattutto con il suo studio innovativo sulle «coalizioni minime vincenti» e a molti altri ambiti di ricerca. Infatti, secondo un recente tentativo di costruire una galleria di studiosi illustri della scienza politica per il periodo 1954-1994, Riker si posiziona ai vertici in base ad almeno due criteri. Nel periodo considerato ha infatti pubblicato ben 16 articoli sull'American Political Science Review ed è stato tra gli autori maggiormente citati sulle più importanti riviste di scienza politica (Miller, Tien e Peebler 1996). Riker non si distingue solo nei giudizi degli scienziati politici americani. Nel volume The New Handbook of Political Science, curato da R.E. Goodin e H.D. Klingemann, Riker è indicato come uno tra coloro che hanno contribuito all'integrazione della scienza politica mondiale ed è stato citato 24 volte, mentre Wheare una sola ed Elazar nessuna (Goodin e Klingemann 1996, 40-41).

Certamente uno dei temi principali del lavoro di Riker è stato il federalismo. Per trent'anni egli è stato il punto di riferimento in questo campo. Nel 1964 ha scritto Federalism: Origin, Operation, Significance, incentrato sul caso americano, ma che tratta, seppure brevemente, tutti i sistemi federali contemporanei (Riker 1964). Nel 1974 ha contribuito, con un lungo e ben argomentato articolo, all'ormai classico lavoro in otto volumi curato da Greenstein e Polsby (1975). Nel 1987 ha riunito la

ra le prime elezioni competitive venissero tenute a livello locale e non a livello nazionale. Al contrario, la sequenza seguita in Spagna per cui si sono tenute prima le elezioni nazionali, seguite dalla formazione di una Assemblea costituente e dalla negoziazione degli Statuti di autonomia, rappresenterebbe un solido sentiero per la costruzione di un sistema democratico federale in Indonesia e in Cina.

11 Il lavoro più importante di Wheare è Federal Government, pubblicato nel 1946 e ristampato nel 1990. Quanto ad Elazar, egli ha pubblicato una quindicina di libri riguardanti il federalismo. Da un punto di vista politologico il suo libro più importante è Exploring Federalism (1987). 
maggioranza dei suoi scritti sul federalismo americano nel libro The Development of American Federalism (Riker 1987a). Nel 1995, poco prima della sua morte, gli fu chiesto, in quanto esponente di primo piano dell'approccio comparato sul federalismo, di scrivere una introduzione teorica per un volume di studi europei sul futuro federale dell'Unione europea (Riker 1996). Come abbiamo detto il pensiero di Riker sul federalismo è direttamente collegato alla sua preoccupazione, sia normativa che empirica, di come evitare i pericoli del populismo democratico. Da questo punto di vista, le teorie di Riker della scelta individuale e della democrazia si fondono in Liberalism against Populism: A Confrontation between the Theory of Democracy and the Theory of Social Choice (1982).

Data la centralità di Riker nella moderna scienza politica e la centralità del federalismo nelle sue argomentazioni concettuali e normative, qualsiasi rivisitazione del federalismo deve fare $\mathrm{i}$ conti con la tradizione rikeriana. Spero di avere già contribuito a sollevare dei dubbi su due dei capisaldi della teoria rikeriana sul federalismo, ovvero: il federalismo produce sempre delle conseguenze normative positive dal punto di vista della teoria democratica; tutti i sistemi federali stabili si basano su di una storia e una logica di accordo del tipo «diventare uniti». $\mathrm{Ci}$ sono altri due punti rilevanti della teoria rikeriana sul federalismo che devo affrontare se voglio procedere oltre nel mio ragionamento. In primo luogo, Riker sostiene che la struttura fondamentale del federalismo moderno è quella che egli chiama «federalismo centralizzato» (centralized federalism). Riker non solo considera gli Stati Uniti come l'origine del suo modello, ma anche come la forma modale di federalismo. Io sostengo che è empiricamente molto utile da un punto di vista comparato poter invece considerare il federalismo democratico come un continuum che va da sistemi poco demo-vincolanti a sistemi molto demo-vincolanti. Alla luce di questo approccio alternativo, gli Stati Uniti emergono non più come il tipo modale del federalismo moderno, ma come un caso eccentrico collocato vicino al polo demo-vincolante del continuum. La seconda argomentazione di Riker è paradossale. Nonostante la sua propensione normativa in favore del federalismo fondata sulla tendenza a frammentare i partiti, egli sostiene che le istituzioni federali di per sé non hanno un impatto sulla politica pubblica. In questo lavoro sostengo, al contrario, che le istituzioni federali contano e contano molto. 
Il federalismo come continuum demo-vincolante

Fino alla Convenzione di Filadelfia del 1787 la sola forma di federalismo nel mondo, secondo Riker, era il «federalismo decentrato» (decentralized federalism). Riker sostiene che nel 1787 i padri fondatori «inventarono il federalismo centralizzato» (1987b). Attraverso i suoi scritti egli sottolinea che «la forma di federalismo che il mondo trova più attraente è quella centralizzata» (Riker 1975, 10). Per Riker tutti i sistemi federali moderni derivano da quello americano, che egli presenta spesso come la norma.

Riker spinge il lettore ad accettare l'implicita dicotomia «federalismo decentrato» versus «federalismo centralizzato». In questa sede, invece, ciò che Riker chiama federalismo decentrato è concepito come una varietà di «alleanze» o di «confederazioni», la cui caratteristica fondamentale è il fatto che la sovranità di ogni membro dell'alleanza o della confederazione non è vincolata costituzionalmente. Occasionalmente i membri potevano essere sottoposti a vincoli, ma solo nella misura in cui ogni membro sovrano giudicava che era nel proprio interesse agire in modo collettivo. In alcuni casi, prima della Convenzione di Filadelfia, quello che Riker chiama «federalismo decentrato» richiedeva un voto unanime. $\mathrm{Ma}$, dal punto di vista analitico, una decisione che richiede l'unanimità è coerente con la sovranità assoluta dei membri individuali dal momento che non può essere presa nessuna decisione contraria ai loro interessi.

Riker ha ragione quando afferma che dalla Convenzione di Filadelfia emerse qualcosa di nuovo. Quello che scaturì fu una formula per vincolare costituzionalmente tutte le unità della federazione in modo tale da rendere l'exit costituzionalmente impossibile o molto difficile. Inoltre l'unanimità non venne più richiesta per le decisioni obbligatorie. Se c'è una dicotomia, allora, questa è tra alleanze e confederazioni da un lato e sistemi federali dall'altro. Se il lettore accetta questa revisione della dicotomia di Riker, possiamo procedere oltre.

Tutti i sistemi federali democratici, in quanto federazioni, vincolano il centro. Invece di concepire una dicotomia tra «federalismo centralizzato» e «federalismo decentrato», ritengo analiticamente più proficuo concepire il federalismo democratico come un continuum che va da un estremo altamente demovincolante ad uno demo-emancipante. L'impiego di un continuum apre la categoria analitica e storica del federalismo ad un in- 
sieme di distinzioni empiriche e concettuali che non sarebbero possibili collocando semplicemente tutti i sistemi federali dentro la singola categoria del «federalismo centralizzato». Per esempio, intendo dimostrare che il modello di federalismo centralizzato degli Stati Uniti più che la norma rappresenta un caso estremo, collocato sul polo demo-vincolante del continuum che mi accingo a costruire. Come dimostrerò in seguito, fra i principali sistemi federali del mondo solo il Brasile presenta infatti una capacità di veto della maggioranza democratica al livello di governo centrale paragonabile a quella degli Stati Uniti. La Germania, che Wheare ha escluso dalla categoria dei sistemi federali in quanto deviante rispetto al modello americano, è in realtà molto più vicina al centro (la norma) del continuum federale. L'India, la cui sopravvivenza come sistema democratico non può essere capita senza analizzarne la componente federale, ma che Wheare ha definito solo «quasi federale», in quanto molto diversa dagli Stati Uniti, si colloca all'estremo meno demo-vincolante del continuum, molto lontano dagli Stati Uniti. Nei termini di questa discussione, l'India negli anni '90 condivide con gli Stati Uniti solo due aspetti: l'essere un paese federale e democratico.

Perché tutti i sistemi federali democratici sono intrinsencamente vincolanti per il centro? Vi sono quattro ragioni. La prima è concettuale e costituzionale, ed ha conseguenze empiriche. La definizione di Dahl cattura sinteticamente la dimensione duale della sovranità propria del federalismo, vale a dire che esso costituzionalmente vincola il demos al livello centrale (Dahl 1986, 114). Se si accoglie la definizione di Dahl, si deve accettare che tutte le federazioni democratiche, in quanto federazioni, vincolano il centro e, almeno in qualche misura, sono anche demo-vincolanti. Si consideri l'impatto del federalismo sull'agenda politica. I sistemi democratici solitamente hanno una agenda aperta. Questo significa che, all'interno delle formule prescritte dalla Costituzione, non ci sono aree di policy in cui la maggioranza democratica non può legiferare. Questa è una delle ragioni per cui Przeworski definisce la democrazia come una forma di «incertezza istituzionalizzata» (1986). Tuttavia, diversamente da un sistema democratico unitario, in tutti i sistemi federali il demos a livello centrale deve accettare una agenda chiusa in quanto alcuni ambiti sono costituzionalmente preclusi ai suoi poteri legislativi (Dahl 1989).

La seconda ragione per cui i sistemi federali democratici vin- 
colano il demos è rappresentata dal fatto che esso è per sua natura suddiviso non solo in tanti demoi, ma anche in varie strutture di autorità. Il demos è vincolato verticalmente come Dahl indica con chiarezza. Inoltre esso è anche vincolato orizzontalmente. A livello centrale ci sono due organi legislativi. Il primo, la Camera bassa, rappresenta il principio della popolazione e può avvicinarsi alla pura eguaglianza democratica («una persona, un voto»). Il secondo, la Camera alta, rappresenta invece il principio del territorio. L'ideologia dell'uguaglianza territoriale (una unità, un voto) può mascherare una grandissima diseguaglianza (il voto di un cittadino in uno stato di piccole dimensioni può valere più di cento voti in uno stato di grandi dimensioni).

La terza ragione per cui i sistemi federali democratici vincolano il demos è collegata alla Costituzione. Tutte le costituzioni democratiche dovrebbero essere «auto-vincolanti» in quanto, nel migliore dei casi, dovrebbero risultare difficili da modificare (Elster e Slagstad 1988). Nel caso estremo di una costituzione molto difficile da modificare ci potremmo chiedere se sia giusto che una generazione democratica possa impegnare per sempre le generazioni democratiche future. Tanto più numerosi sono gli ambiti che costituzionalmente si è stabilito debbano restare fuori dalla portata del livello centrale democratico di un sistema federale, tanto più numerosi saranno gli ambiti di policy in cui al livello centrale le generazioni future, per essere in grado di promulgare le leggi, dovranno produrre delle super-maggioranze. Ipoteticamente, potrebbe verificarsi che ad un certo punto, in futuro, una schiacciante maggioranza di cittadini in tutte le subunità voglia diventare uno stato unitario. Eppure, la generazione che ha definito la Costituzione potrebbe avere esplicitamente precluso qualsiasi emendamento che possa modificare la natura fondamentale del sistema federale. In tal caso la sola possibilità rimarrebbe quella di scrivere una nuova costituzione.

La maggior parte dei cambiamenti politici non sono così estremi come nel caso ipotetico ora suggerito, tuttavia modificare le regole del gioco di un sistema federale rimane estremamente difficile. Dalla prospettiva della teoria dei giochi come da quella del comportamento legislativo, le regole decisionali più difficili da modificare sono quelle che strutturalmente favoriscono un gruppo il cui voto favorevole è tuttavia necessario per cambiare le regole stesse. Un voto nella Camera alta che renda meno demo-vincolante un sistema federale altamente iniquo identifica uno scenario di questo tipo. 
La quarta ragione per cui i sistemi federali democratici sono demo-vincolanti deriva dalle prime tre. Le Costituzioni dei sistemi democratici federali sono necessariamente più complesse di quelle dei sistemi democratici unitari. Potenziali questioni di policy in aree come l'ambiente, il welfare, la legislazione, la sanità, la ricerca devono essere costantemente ridefinite socialmente ed economicamente. I confini di ciò che è unanimemente di competenza del governo della sub-unità o del governo nazionale sono costantemente mutevoli. La definizione dei confini è pertanto più importante e più difficile in un sistema federale che in uno unitario. Infatti, un altro importante attore politico che non deriva il suo potere di decisione da un controllo periodico da parte del demos o persino dei demoi - il potere giudiziario - gioca di solito in un sistema federale un ruolo più rilevante che in un sistema unitario.

Per queste ragioni i sistemi democratici federali sono tutti intrinsecamente demo-vincolanti. Tuttavia, come dimostrerò in seguito, possono diversificarsi $\mathrm{e}$ in realtà si diversificano in modo consistente rispetto al grado di limitazione che esercitano sul demos. Infatti, vi sono sistemi federali che sono costruiti in modo da conferire potere alla cittadinanza, pur nei limiti del federalismo. La debolezza analitica della dicotomia proposta da Riker tra «federalismo decentrato» e «federalismo centralizzato» deriva dal fatto che essa rende molto difficile cogliere e valutare l'immensa variabilità all'interno della categoria del «federalismo centralizzato». In seguito cercherò di operazionalizzare il mio concetto di continuum federale e di dimostrare come sia in grado di aprire nuove strade per l'analisi comparata, empirica e normativa, del federalismo.

\section{Federalismo e status quo strutturale}

L'ultima tesi di Riker sul federalismo che dobbiamo valutare riguarda il processo di policy-making. Riker considera le preferenze individuali come la forza trainante della scelta sociale. Partendo da questa premessa, egli sostiene che se un insieme di individui ritiene che un assetto istituzionale come quello federale determina policies sgradite, sia per loro relativamente facile modificare sia le istituzioni che le politiche. Questa argomentazione ha fatto di Riker lo studioso più autorevole del federalismo, ma, al contempo, l'ha spinto ad affermare che l'oggetto 
della sua ricerca scientifica è in fondo una chimera senza potere. Lasciatemi citare alcune delle sue parole sul perché le istituzioni federali, a suo avviso, non contano:

È difficile evitare di giungere alla conclusione che gli accidenti del federalismo (ossia i dettagli costituzionali e amministrativi) non facciano alcuna differenza. Essi forniscono semplicemente uno standard di stile per i paesi federali che si differenzia sotto certi aspetti dallo standard dei paesi unitari. Nei paesi federali è spesso necessario dimostrare che un governo ha il potere legale di fare ciò che vuole. Ma se realmente lo vuole fare, ha sempre il potere per farlo. I giuristi, soprattutto quelli costituzionali, hanno un po' più da lavorare in un sistema federale che in uno unitario, per il resto non c'è molta differenza (Riker 1975, 144).

Indipendentemente da quanto utile sia la finzione del federalismo nel creare un nuovo governo, non si dovrebbe mai mettere in ombra il fatto che è una finzione. Nello studio dei governi federali, pertanto, è sempre appropriato andare oltre la finzione per studiare le forze reali di un sistema politico (Riker 1969, 146).

Quello che conta non è la struttura costituzionale, per altro irrilevante, ma piuttosto la cultura politica ed economica... Il federalismo è al massimo una variabile interveniente e relativamente poco importante (Riker 1969, 144-145).

Il federalismo non fa particolare differenza a livello di politica pubblica (Riker 1975, 143).

Una delle ragioni per la strana morte del federalismo nella moderna teoria democratica è dovuta al fatto che è stato il principale teorico del federalismo ad ucciderlo. Il ragionamento che sta dietro i giudizi di Riker è che egli vede le decisioni collettive e quelle politiche come derivanti fondamentalmente da un aggregato di preferenze individuali. In modo stilizzato potremmo dire che egli considera le preferenze aggregate come inerentemente instabili ed essenzialmente incoerenti. La democrazia incarna un «disequilibrio indotto dalle preferenze». Le preferenze determinano le domande. Le istituzioni traducono le preferenze in politiche. Pertanto sono le preferenze la spiegazione fondamentale dei risultati di policy. $\mathrm{E}$ in base a questa logica che Riker afferma che le istituzioni federali sono «al massimo una variabile interveniente e relativamente poco importante».

In realtà, in alcuni dei suoi lavori Riker afferma che le istituzioni possono contare, ma il nocciolo della sua argomentazione è che le preferenze contano più delle istituzioni. Per Riker «le istituzioni sono probabilmente meglio identificabili come 
preferenze congelate... Se le istituzioni sono preferenze congelate e le preferenze non sono equilibrate, allora anche le istituzioni, eccetto che per eventi di breve periodo, lo sono» (Riker 1980, 445 , corsivo aggiunto).

Le preferenze contano, ma anche le istituzioni. In alcuni casi una democrazia può crollare o scivolare nella guerra civile nel giro di poco tempo. Un esempio lo si trova nella storia americana: nel breve periodo (vale a dire nei vent'anni che hanno portato al primo sparo) il federalismo americano altamente demo-vincolante ha contribuito allo scoppio della guerra civile.

Come Shepsle e Weingast sostengono in modo convincente, parte della forza dell'argomentazione di Riker deriva dalla duplice assunzione che gli individui non hanno preferenze o agende stabili e che le istituzioni in cui essi vivono non hanno contesti vincolati che strutturino gli incentivi ${ }^{12}$. Se queste due affermazioni tengono, allora possiamo affermare che, se l'insieme degli individui in una legislatura manifesta una preferenza per una data politica, essi possono facilmente approvare leggi rilevanti. Tuttavia, come Shepsle e Weingast dimostrano, nessuna di queste ipotesi è confermata dagli studi sul legislativo. I due autori mostrano, per esempio, come persino nella Camera dei rappresentanti americana questioni istituzionali come le routine burocratiche, le regole decisionali, l'anzianità in importanti commissioni e la regola che ogni legge approvata debba, nel giudizio dei controllori, essere superiore allo status quo, spesso producono un «equilibrio strutturalmente indotto». Shepsle e Weingast dimostrano come sistemi decisionali strutturati istituzionalmente possono avere un forte impatto sulla politica pubblica. Lo status quo spesso prevale non a causa delle preferenze individuali ma a causa delle specifiche strutture istituzionali del Congresso e anche perché i deputati entrano a far parte di questo assetto istituzionale non da contesti completamente privi di vincoli ma al contrario da contesti a loro volta vincolati. Il contesto generale dentro cui i singoli deputati di fatto operano ha a che fare con i gruppi di interesse regionali che li eleggono e che decideranno della loro sconfitta o della loro rielezione nel giro di due anni. Correttamente intesi, i vettori del nuovo istituzionalismo e i vettori della scelta razionale individuale, nel caso dei sistemi federali, potrebbero puntare nella stessa direzione, ossia

12 Su come le istituzioni possono strutturare e ristrutturare gli incentivi si vedano $\mathrm{i}$ pioneristici articoli di Shepsle e Weingast $(1981 ; 1987)$. 
verso lo status quo. Se questo può accadere nella Camera bassa americana, che è prossima alla rappresentanza proporzionale in termini di «una persona un voto», non è necessario essere un «neo istituzionalista» per cogliere le implicazioni di policy del federalismo in un paese come il Brasile, che si colloca, in virtù delle prerogative del Senato, al polo estremo del continuum demo-vincolante e dove le constituencies e i governatori che contribuiscono ad eleggere i membri al Senato hanno la loro agenda e le loro risorse di controllo che i senatori valutano ${ }^{13}$. Il Brasile è una nuova democrazia che combatte con una delle più inique distribuzioni di reddito del mondo. Nel 1996 la maggioranza della popolazione espresse in modo continuativo chiare preferenze per le riforme e il Presidente Cardoso, che aveva in prima persona sostenuto le riforme, terminò l'anno con un sostegno estremamente alto. Tuttavia, né le preferenze dei cittadini né quelle del Presidente furono sufficienti alla realizzazione delle riforme. Nella Camera alta brasiliana un gruppo di senatori che rappresentano meno del $9 \%$ dell'elettorato sono in grado di produrre un «gruppo vincente» per bloccare una riforma legislativa. Se le istituzioni della Camera dei rappresentanti americana, equamente rappresentativa della popolazione (apportioned) sono in grado di produrre equilibri strutturalmente indotti, il federalismo brasiliano, che ha sia una Camera alta che una Camera bassa assai poco rappresentative della popolazione ( $m a$ lapportioned), può certamente contribuire al perpetuarsi di uno status quo strutturalmente indotto. Nessuno studioso del Brasile, dopo avere attentamente analizzato le conseguenze delle regole decisionali e delle prerogative del Senato, degli Stati e dei governatori, potrebbe sostenere che il federalismo è una variabile interveniente relativamente poco importante.

13 David Fleischer (professore di Scienza Politica all'Università di Brasilia) ha stimato che quasi il $40 \%$ dei senatori federali brasiliani sono stati governatori (molti più di una volta) e che molti dei senatori aspirano a diventarlo. Quasi nessun senatore americano è stato governatore $e$ ambisce a diventarlo. Per avere valide prove di come il percorso di carriera dei deputati e dei senatori in Brasile sia fortemente orientato e influenzato dagli stati da cui provengono, cfr. Mainwaring e Samuels (1997) e Abruçio e Samuels (1997). Viene stimato che nella legislatura 1991-94 circa il $35 \%$ dei deputati eletti hanno dichiarato di preferire o di fatto hanno rinunciato al loro seggio per ottenere un incarico legislativo o di governo a livello statale. 


\section{L'operazionalizzazione del continuum demo-vincolante}

Dopo aver sollevato questioni empiriche e normative sul pensiero di Riker, è ora il momento di tentare di operazionalizzare il concetto di continuum di vincoli per il demos e impiegarlo per l'analisi comparata della politica nei sistemi federali.

I sistemi democratici federali possono variare in modo significativo riguardo alle pratiche e formule decisionali inserite nella Costituzione che vanno contro il principio democratico «una persona, un voto». $\mathrm{Ci}$ possono anche essere modelli para-costituzionali e di comportamento politico estremamente importanti, in grado di mitigare o esacerbare i limiti della capacità di una maggioranza nazionale di attuare le politiche. Mentre ognuno dei fattori individuali sopra menzionati ha un impatto sulle capacità legislative del demos, quello che è davvero importante è come questi fattori interagiscono tra loro in modo da impedire sistematicamente la capacità di una maggioranza potenziale di alterare lo status quo o in modo da facilitare (nei limiti delle aree dei diritti delle sub-unità garantiti costituzionalmente) la capacità di una maggioranza di approvare decisioni generali che si ritengono necessarie per la qualità della democrazia e per un processo decisionale efficace. In questo articolo farò particolare attenzione a quattro variabili, tre delle quali costituzionali mentre una, la più importante, è una pratica para-costituzionale dei partiti politici. Le quattro variabili e le ipotesi riguardanti il loro rispettivo potenziale demo-vincolante sono le seguenti:

1) Grado di sovrarappresentanza nella Camera territoriale: maggiore è il grado di sovrarappresentanza degli Stati meno popolati (e maggiore, quindi, la sotto-rappresentanza di quelli più popolati), maggiore è il potenziale demo-vincolante del Senato.

2) Prerogative di policy (policy scope) della Camera territoriale: maggiori le prerogative di policy della Camera che incarna il principio di territorialità, maggiore il potenziale di limitazione dei poteri legislativi della Camera che rappresenta il principio della popolazione.

3) Quantum di attribuzione del policy making alle sotto-unità della federazione sancito dalla Costituzione: maggiore il numero di competenze decisionali costituzionalmente sottratte al governo centrale, maggiori i vincoli per il demos.

4) Grado in cui il sistema partitico, nel suo orientamento e per i suoi sistemi di incentivi, si presenta nazionalizzato (politywide): più i partiti politici sono partiti disciplinati e i loro siste- 
mi di incentivi - soprattutto quelli relativi ai processi di selezione - privilegiano gli interessi nazionali su quelli provinciali e locali, più tali partiti sono in grado di mitigare le caratteristiche demo-vincolanti proprie del federalismo.

Grado di sovrarappresentanza nella Camera territoriale. Tutti i sistemi federali hanno una Camera legislativa che rappresenta le sotto-unità che costituiscono la federazione (da qui in avanti chiamata Senato, Camera territoriale o Camera alta) e una $\mathrm{Ca}$ mera legislativa che rappresenta la popolazione (da qui in avanti Camera bassa). Dal punto di vista del principio democratico «una persona un voto», tutte le Camere alte violano in qualche modo il principio democratico di uguaglianza. Tuttavia, ciò che spesso non viene riconosciuto è che ci possono essere, e in realtà ci sono, enormi variazioni nel grado di diseguaglianza secondo cui i piccoli stati sono sovrarappresentati nelle Camere alte degli stati federali.

L'esempio più conosciuto e il modello più emulato di federalismo democratico è quello che si costituì con la Convenzione di Filadelfia del 1787. Ma il modello americano ha varie caratteristiche che non sono essenziali per il federalismo democratico. Infatti, si potrebbe sostenere che il modello americano, in quanto modello, dovrebbe essere sottoposto a dibattito ed essere oggetto di negoziati e sfide. Una caratteristica problematica del modello americano riguarda la composizione della Camera alta.

Il fondamento logico delle Camere alte nei sistemi federali sta nel fatto che esse devono occuparsi di questioni particolarmente rilevanti per le sotto-unità della federazione. Non in base ad un principio, ma piuttosto come conseguenza di uno storico «grande compromesso» tra i grandi e i piccoli stati, nel 17871788 i rappresentanti dei grandi stati fecero due concessioni che violarono l'uguaglianza democratica formale ${ }^{14}$. In primo luogo, attribuirono ai piccoli stati una rappresentanza uguale o, per meglio dire, una enorme sovrarappresentanza nella Camera alta. In secondo luogo, con scarsa consapevolezza delle conseguenze che ne sarebbero derivate, equipararono le prerogative di policy delle due Camere. Queste due decisioni che io definisco «rappresentanza non proporzionale» (disproportionate representation) e «simmetria nelle prerogative di policy» (symmetrical

14 Per un'ottima discussione su come e perché si è arrivati a questi compromessi, cfr. Swift (1996) e Riker (1987a). 
policy scope) costituiscono una parte fondamentale della formula federale degli Stati Uniti. Ma devono per questo essere necessariamente parte integrante di qualsiasi federalismo democratico moderno? Concentriamo per ora la nostra attenzione sulla prima variabile, la composizione della Camera alta.

All'interno dei sistemi federali democratici esistenti alcuni stati sono sovrarappresentati per un rapporto maggiore di uno a cento. Questa enorme sovrarappresentanza non è una caratteristica necessaria del federalismo democratico. Se una minoranza etnica o culturale fosse drasticamente sovrarappresentata nella Camera alta e se quest'ultima avesse prerogative di policy analoghe a quelle della Camera democraticamente eletta, ciò creerebbe senza dubbio, a livello centrale, problemi di lealtà verso la federazione da parte di alcuni leader, soprattutto nel caso di una federazione multinazionale.

Quanto è grande la variabilità della sovrarappresentanza nei moderni sistemi democratici federali? Il sistema federale con il più basso grado di sovrarappresentanza è il Belgio, con un coefficiente di rappresentanza dell'indice di diseguaglianza di Gini di appena $0,015^{15}$. L'India ha 0,10, la Germania 0,32. Gli Stati Uniti hanno un coefficiente di sovrarappresentanza di Gini nella seconda Camera pari a 0,49 e il Brasile prossimo a 0,52.

Quali sono i modelli empirici dietro tali differenze nel coefficiente di diseguaglianza di Gini nella seconda Camera? In Austria, che è solo leggermente meno proporzionale del Belgio, ci sono ad esempio 9 Länder. Ognuno di essi è rappresentato nella Camera federale, ma il numero dei rappresentanti rispecchia da vicino la grandezza demografica di ciascuno: 12 seggi per Vienna, 12 per l'Austria inferiore, 10 per l'Austria superiore, 4 per la Carinzia, 5 per il Tirolo, 3 per Burgenland e altrettanti per Voralberg (Europa World Year Book 1990, 405).

In Germania la Legge fondamentale del 1949 stabilì che le sotto-unità della federazione o Länder avrebbero avuto tra i 3 e i 6 voti nella Camera alta (Bundesrat). «Ogni Land deve avere almeno tre voti; i Länder con più di due milioni di abitanti ne hanno 4, i Länder con più di sei milioni di abitanti 5 e i Länder con più di sette milioni di abitanti 6» (così l'art. 51 della Costi-

15 L'indice di diseguaglianza di Gini misura il grado di diseguaglianza tra variabili. L'indice pari a 0 indica piena uguaglianza mentre pari a 1 indica il massimo di diseguaglianza (che significherebbe in questo caso che un solo stato ha tutti i rappresentanti). Cfr. Lijphart (1984, 173-175). 
tuzione di Bonn; cfr. Finer, Bogdanor e Rudden 1995, 150). Il Land più piccolo, Brema, nel 1993 aveva una popolazione di 686.000 persone ed aveva diritto a 3 voti nella Camera alta. Il Land più grande, Westfalia-Reno settentrionale aveva una popolazione di 17.679 .000 e aveva diritto a 6 voti nella Camera alta (Europa World Year Book 1995, 1293). Pertanto un voto a Brema valeva 13 voti in Westfalia-Reno settentrionale.

Gli Stati Uniti e il Brasile hanno regole decisionali uguali riguardo ai voti nella Camera alta. Ogni stato, indipendentemente dalla popolazione, riceve un uguale numero di seggi in Senato (2 negli Stati Uniti e 3 in Brasile). Negli Stati Uniti, nel 1990, lo Stato meno popolato era il Wyoming con 453.588 abitanti, quello con il maggior numero di abitanti era invece la California con 29.760.021 (Whitacker's Almanack 1997). Un voto in Wyoming valeva quindi 66 voti in California. Il Brasile è ancora più demo-vincolante degli Stati Uniti: nel 1991, l'unità territoriale meno popolata, lo Stato di Roraima, contava 215.790 residenti; quella più grande, San Paolo, 31.192 .818 (Europa World Year Book 1995, 618; Elazar 1994, 44). Un voto in Roraima ha quindi 144 volte il peso di un voto dato a San Paolo.

La natura demo-vincolante del federalismo brasiliano risulta ancora più estrema se consideriamo la Camera bassa. Per ragioni di spazio non tratterò della rappresentanza nella Camera bassa come una variabile a sé. Tuttavia voglio sottolineare che il potere retorico e politico del bisogno di rappresentare il territorio (in quanto opposto alla rappresentanza della popolazione e al principio «una persona un voto») è così forte in Brasile che ogni Stato, per quanto demograficamente piccolo, riceve un minimo di 8 deputati nella Camera dei rappresentanti, mentre non può superare in nessun caso il tetto massimo di 70 deputati. Se ci fosse perfetta proporzionalità, Roraima avrebbe un deputato e San Paolo quasi 115; in realtà Roraima ne conta 8 e San Paolo solo $70^{16}$. In base alla prima variabile, quindi, Belgio, Austria e India si collocano sul versante negativo del continuum demovincolante, la Germania si colloca in posizione intermedia, Stati Uniti, Brasile e Argentina si caratterizzano come $i$ paesi più demo-vincolanti (tab. 1).

16 Per una discussione su questo grave problema di cattiva distribuzione dei seggi nella Camera bassa si veda l'eccellente lavoro di Mainwaring (1998). La stima di 115 deputati per San Paolo nel 1997 è fatta dal Prof. Fleischer (Università di Brasilia). 
TAB. 1. Grado di sovra-rappresentanza della Camera alta in 12 democrazie federali 1

\begin{tabular}{|c|c|c|c|c|c|c|c|c|c|c|c|c|}
\hline $\begin{array}{l}\text { Indice di } \\
\text { diseguaglianza } \\
\text { di Gini }^{2}\end{array}$ & $\begin{array}{c}\text { Belgio } \\
.015\end{array}$ & $\begin{array}{c}\text { Austria } \\
.05\end{array}$ & $\begin{array}{c}\text { India } \\
.10\end{array}$ & $\begin{array}{c}\text { Spagna } \\
.31\end{array}$ & $\begin{array}{c}\text { Germania } \\
\quad .32\end{array}$ & $\begin{array}{c}\text { Canada } \\
.34\end{array}$ & $\begin{array}{c}\text { Australia } \\
.36\end{array}$ & $\begin{array}{c}\text { Russia }^{3} \\
.43\end{array}$ & $\begin{array}{c}\text { Svizzera } \\
.45\end{array}$ & $\begin{array}{l}\text { UsA } \\
.49\end{array}$ & $\begin{array}{c}\text { Brasile } \\
.52\end{array}$ & $\begin{array}{c}\text { Argentina } \\
.61\end{array}$ \\
\hline $\begin{array}{l}\text { Rapporto tra } \\
\text { l'unità federale } \\
\text { meglio e peggio } \\
\text { rappresentata } \\
\text { (sulla base della } \\
\text { popolazione) }\end{array}$ & $\begin{array}{c}\text { Austria } \\
1.5 / 1\end{array}$ & $\begin{array}{c}\text { Belgio } \\
2 / 1\end{array}$ & $\begin{array}{c}\text { Spagna } \\
10 / 1\end{array}$ & $\begin{array}{c}\text { India } \\
11 / 1\end{array}$ & $\begin{array}{c}\text { Germania } \\
13 / 1\end{array}$ & $\begin{array}{c}\text { Australia } \\
13 / 1\end{array}$ & $\begin{array}{c}\text { Canada } \\
21 / 1\end{array}$ & $\begin{array}{c}\text { Svizzera } \\
40 / 1\end{array}$ & $\begin{array}{l}\text { UsA } \\
66 / 1\end{array}$ & $\begin{array}{c}\text { Argentina } \\
85 / 1\end{array}$ & $\begin{array}{c}\text { Brasile } \\
144 / 1\end{array}$ & $\begin{array}{l}\text { Russia } \\
370 / 1\end{array}$ \\
\hline
\end{tabular}

1 Informazioni complete su tutti i paesi federali sono contenute nell'appendice del database di Stepan-Swenden sul federalismo. Siamo grati a Cindy Skach e Jeff Kahn per averci fornito, rispettivamente, i dati sull'India e la Russia. Gli altri dati sono stati presi da Whitaker's Almanack (1997), The Europa World Year Book (1995), Elazar (1994). Sulle disposizioni costituzionali relative alle Camere alte cfr. Finer, Bogdanor e Rudden (1995) e Blaustein e Flanz (1991).

2 La formula è: $G=1+1 / n-2 n^{2} y(y 1+2 y 2+3 y 3+\ldots+n y n)$, dove $n=$ numero di unità, $y=\%$ media dei seggi. Il coefficiente di Gini è uguale a zero se la composizione della Camera alta è pienamente proporzionale mentre è uguale a uno se una sotto-unità dispone di tutti $i$ voti alla seconda Camera. Lijphart è stato uno dei primi ad usare il coefficiente di Gini per misurare la disuguaglianza nella composizione della seconda Camera (cfr. Lijphart 1984, 174).

3 Lo status della Russia come democrazia è quello più discutibile tra i 12 paesi della tabella. I dati sono stati inclusi per scopi di comparazione. 
Prerogative di policy della Camera territoriale. Un sistema federale ha senso solo se le sotto-unità hanno un qualche ruolo nell'approvare o almeno nell'emendare le leggi che direttamente incidono sul funzionamento della federazione. Ciò è particolarmente vero per i sistemi federali multinazionali e multiculturali. I poteri legislativi riguardanti questioni culturali come la lingua, la religione o l'educazione rappresentano una delle ragioni di fondo dell'esistenza di sistemi federali multinazionali.

Tuttavia, i sistemi legislativi federali differiscono grandemente per quanto riguarda le competenze federali attribuite alla Camera alta. La Camera dei rappresentanti americana ha prerogative maggiori del Senato nelle leggi riguardanti questioni finanziarie e pertanto alcuni studiosi ritengono che abbia più poteri di esso. Vi sono tuttavia ambiti nei quali il Senato, pur essendo meno rappresentativo, ha più poteri della Camera dei rappresentanti (che è ridefinita proporzionalmente ogni decennio in linea con i cambiamenti demografici). $\grave{E}$ il caso della formazione della Corte suprema: il Presidente ne nomina i membri, il Senato conferma o respinge le nomine del Presidente, la Camera dei rappresentanti si trova in una posizione marginale. Lo stesso vale per tutte le principali cariche di governo (Segretario, Vice-Segretario, assistenti del Segretario di tutti i più importanti Dipartimenti), nonché per le posizioni di vertice della Central Intellingence Agency, del National Security Council, del Federal Bureau of Investigation e di molte altre agenzie governative, che devono essere anch'esse confermate dal Senato.

Il Brasile in teoria ha adottato la formula costituzionale americana della simmetria di potere tra le due camere. $\mathrm{La} \mathrm{Ca-}$ mera bassa, come negli Stati Uniti, ha maggiore potere nelle questioni finanziarie. Inoltre, a meno che il Senato non voti contro un intero provvedimento, la Camera dei rappresentanti può modificare qualsiasi emendamento del Senato senza interpellarlo. In 12 aree, tuttavia, la competenza del Senato è esclusiva. Il Senato, ad esempio, nomina direttamente due terzi dei giudici che controllano le spese federali e ha il diritto di rigettare o confermare l'altro terzo; ha una competenza esclusiva in tema di indebitamento pubblico e può scavalcare un parere negativo della Banca centrale; ha competenza esclusiva in tema di indebitamento estero. In Brasile nessuna area di policy è sottratta alle competenze del Senato, mentre alcune policy-chiave sono di esclusiva prerogativa del Senato.

In Brasile la relazione tra le due variabili implica che gli sta- 
ti che rappresentano solo il $13 \%$ degli elettori hanno il $51 \%$ dei voti in Senato. Questo piccolo gruppo di senatori deve pertanto essere trattato con la deferenza, il patronage e lo scambio di favori dovuto a un gruppo che può, teoricamente, bloccare l'attuazione delle politiche sostenute dai senatori che rappresentano l'87\% della popolazione.

Il modello americano di «simmetria nelle prerogative di policy» non è, evidentemente, l'unico modello possibile. Esso, ancora una volta, si colloca ad un estremo del continuum demovincolante. Ė importante sottolineare come né la teoria, né la prassi democratica moderna richiedano che una Camera fortemente disproporzionale abbia poteri di attuazione delle politiche pari a quelli della Camera proporzionale. Non vedo, ad esempio, nessun serio problema nel fatto che la seconda Camera in Germania (Bundesrat) sia trattata in modo ingiusto o non democratico a causa delle sue limitate prerogative di policy. Il Bundestag, prossimo alla piena proporzionalità, rappresentando tutti gli elettori tedeschi, ha il potere esclusivo di eleggere e rimuovere il Cancelliere. Il famoso «voto di sfiducia costruttivo» è espresso solo nella Camera bassa. Ugualmente la seconda Camera in origine aveva solo il potere di voto per circa un terzo delle leggi federali, riguardanti direttamente questioni rilevanti per i Länder ${ }^{17}$.

La differenza tra il modello americano e quello che è poi diventato il modello tedesco con riferimento alla variabile «prerogative di policy della Camera territoriale» era ben chiara alle forze di occupazione americane durante la stesura della Costituzione di Bonn, che infatti vi si opposero. Secondo un eminente studioso di istituzioni politiche come Herman Finer, «gli americani erano convinti che una autorità federale debole fosse preferibile. Essi continuarono a sostenere le tesi sul federalismo e sulla divisione dei poteri del nascente Stato americano nel 1787, ovvero che la salvaguardia di un sistema stabile e democratico, per quanto frammentato, richiedesse di mantenere debole il governo. Fu questa la causa del passaggio all'opposizione dell'Spd». Alla fine l'Spd ebbe la meglio sulle obiezioni degli ame-

17 Quasi cinquanta anni dopo la promulgazione della Legge fondamentale, il Bundesrat oggi approva tra il 55 e il $60 \%$ di tutta la legislazione federale. Questo avviene a fini di compensazione per il fatto che il federalismo tedesco è diventato con il tempo più «centralizzato»e «amministrativo». Recentemente i Länder hanno ottenuto il diritto di voto su alcune questioni riguardanti l'integrazione federale nella Unione Europea. 
ricani e riuscì ad ottenere che «la seconda Camera non sarebbe stata dominante rispetto alla prima» (Finer 1956, 690). Il federalismo tedesco, pertanto, è meno simmetrico in termini di prerogative di policy di quello americano. Sulla base di entrambe le dimensioni si può quindi affermare che il federalismo tedesco è formalmente (e, credo, sostantivamente) più democratico ${ }^{18}$.

La Spagna è meno demo-vincolante della Germania. Come in Germania, la Camera bassa (Cortes), ha competenze esclusive sulle due più importanti questioni nazionali, autorizzare la formazione del governo e votare una mozione di «sfiducia costruttiva». La Camera alta, invece, ha un potere di veto sulle leggi approvate dalla Camera bassa inferiore a quello del Bundestag. Il più importante potere della Camera alta spagnola è la capacità di bloccare un intervento armato del governo centrale in una delle Comunità autonome. Dal momento che tale intervento ha una rilevanza diretta sulla natura della federazione, è corretto che la Camera territoriale abbia a questo proposito speciali poteri. La Costituzione spagnola dedica grande attenzione a questo aspetto, come vedremo quando tratteremo della terza variabile.

All'estremo inferiore del continuum demo-vincolante delle Camere territoriali ci sono paesi come l'India, la Spagna e il Belgio. Tali paesi occupano posizioni così estreme e le loro $\mathrm{Ca}$ mere territoriali sono così deboli che si potrebbe persino ritenere di non doverli considerare paesi federali. Tuttavia, se si accetta la definizione di Dahl (secondo la quale la federazione è un sistema nel quale le competenze delle unità locali sono costituzionalmente precluse all'autorità del governo nazionale), Spagna e Belgio sono senza dubbio sistemi federali a causa della terza variabile (di cui si dirà tra breve), relativa al potere delle unità locali, in quei due paesi abbastanza consistente. Swenden chiarisce che uno degli aspetti distintivi del federalismo belga è la sua natura di comunità plurinazionale e multiculturale, che combina tra loro caratteristiche federali e consociative. In base all'indice di Gini, la Camera alta del Belgio risulta nel database di Stepan-Swenden la più prossima alla piena rappresentanza proporzionale. Il Senato belga, tuttavia, deve dare parere favorevole agli emendamenti costituzionali e ai trattati internazionali e a tutte le leggi che riguardano la

18 Sul federalismo tedesco e in particolare sul concetto costituzionale conosciuto come Bundestreue (spesso tradotto come federal trust o comity), cfr. de Villiers (1995). 
struttura delle istituzioni federali e l'organizzazione giudiziaria del paese. Ma gli emendamenti alla Costituzione o le leggi che riguardano la struttura federale o gli statuti linguistici delle Comunità richiedono una maggioranza qualificata. Tutti gli emendamenti costituzionali richiedono una maggioranza dei due terzi in entrambe le Camere. Tutte le leggi o gli emendamenti costituzionali riguardanti questioni linguistiche non solo richiedono la maggioranza parlamentare di due terzi ma anche una maggioranza all'interno di ogni gruppo linguistico in Parlamento. Vale la pena di ricordare che in queste occasioni entrambe le Camere vengono suddivise in base alle linee linguistiche. Alcuni critici hanno sottolineato che non c'è bisogno di un sistema federale fortemente bicamerale dal momento che la tecnica della «maggioranza qualificata» si applica anche alla Camera bassa (Swenden 1997).

In base alla Costituzione, la Camera alta spagnola e quella indiana, così come quella tedesca, non possono esprimere né il voto di fiducia, né il voto di censura. Tuttavia, diversamente dalla Germania, la Camera alta spagnola e indiana non hanno alcuna funzione legislativa importante nel sistema federale ${ }^{19}$. Esse assomigliano infatti ad una Camera di revisione del tipo della Camera dei Lords. Su un importante terreno, tuttavia, la Camera alta spagnola gioca un ruolo cruciale nel preservare l'autonomia delle unità locali. Si tratta del diritto del governo centrale di ricorrere alla forza per imporre l'«ordine» alle sotto-unità. Tutti i sistemi democratici sono dotati di qualche meccanismo per la legislazione di emergenza. La questione fondamentale è se tali meccanismi siano scritti con la chiarezza necessaria da rendere tali poteri di emergenza attivabili unicamente con il consenso del legislativo, per scopi ben definiti approvati dal legislativo e solo per periodi di tempo limitati anch'essi determinati dal legislativo. Poiché un intervento armato del centro contro le sotto-unità riguarda un aspetto fondamentale della federazione in quanto tale, l'attribuzione alla Camera

19 La Camera alta indiana a livello centrale - il Consiglio degli Stati - ha alcuni poteri di veto. Ad esempio, l'art. 249 della Costituzione stabilisce che il Parlamento non può legiferare rispetto a una questione che è stata attribuita alle assemblee legislative degli Stati, a meno che $2 / 3$ dei membri presenti e votanti nel Consiglio degli Stati non voti a favore. L'art. 368 stabilisce che gli emendamenti costituzionali non-possono passare a meno che non vengano approvati da una maggioranza qualificata dei membri del Consiglio degli Stati. L'articolo stabilisce anche che qualsiasi emendamento deve essere ratificato da almeno metà dei sistemi legislativi statali. 
alta di un ruolo centrale a riguardo è assolutamente appropriata.

Interventi federali di questa natura sono discussi nell'art. 155 della Costituzione spagnola. Per poter avere luogo, tali interventi devono soddisfare quattro condizioni. In primo luogo, il governo deve formalizzare in una propria risoluzione l'avvenuta violazione della costituzione o la condotta anticostituzionale in atto da parte di una sotto-unità. In secondo luogo, il governo centrale deve trasmettere questa risoluzione alla sottounità per iscritto, richiedendo una risposta formale in merito. Terzo, se il governo ritiene inadeguata la risposta ricevuta può domandare alla Camera alta di votare l'intervento federale. Quarto, perché l'intervento possa aver luogo la Camera alta deve esprimersi con il favore della maggioranza assoluta dei propri membri. Questo insieme di procedure consente che vi sia spazio per trattative e mutui aggiustamenti durante l'intero processo. Nei primi 19 anni della Costituzione spagnola non si è verificato nessun intervento in base all'art. 155; credo sia corretto affermare che la Camera alta gioca una funzione appropriata di limitazione del demos contro l'intervento federale.

Anche per l'India è probabilmente corretto affermare che la formula costituzionale riguardante gli interventi federali non vincola in modo sufficiente un governo in possesso della maggioranza. L'art. 356 della Costituzione stabilisce che il Consiglio dei ministri può chiedere un intervento del Presidente (il cosiddetto President's Rule) con cui sciogliere il legislativo e il governo provinciale e porre la provincia sotto il diretto controllo del governo centrale. L'unico requisito per tale intervento è che il governatore (un funzionario non eletto, nominato dal governo centrale) indichi che il governo della provincia «non è più in grado di proseguire nel rispetto delle norme della Costituzione». Per i primi 60 giorni non è richiesto alcun voto, né della Camera alta né della Camera bassa, che autorizzi l'intervento federale. Dopo 2 mesi entrambe le Camere votano un rinnovo per altri 6 mesi oppure l'intervento ha termine. La Camera alta ha pertanto il potere costituzionale di limitare un intervento federale. $\mathrm{Ma}$ le norme costituzionali riguardanti l'intervento sono così vaghe e il Partito del Congresso in passato ha spesso goduto di una tale maggioranza e disciplina di carattere nazionale (quarta variabile) che si è fatto spesso ricorso al President's Rule, persino sotto il governo democratico e consociativo di Nehru. Infatti, tra il 1947 e il 1997 l'intervento del 
Presidente è stato impiegato oltre un centinaio di volte e ha riguardato tutti gli Stati.

Negli ultimi anni, tuttavia, una serie di fattori - tra cui le reiterate richieste indirizzate dalla Corte al governo centrale con l'invito a rendere di pubblico dominio $i$ vari passaggi che avevano motivato l'intervento, il crescente numero di governi di minoranza a livello centrale, il maggior peso coalizionale dei partiti locali provinciali - ha reso il ricorso al President's Rule più difficile e controverso ${ }^{20}$. Se si restringe la nostra analisi ai soli poteri di veto della Camera alta, si può affermare che la dimensione federale della democrazia indiana potrebbe essere migliorata se fosse richiesta una maggioranza assoluta della $\mathrm{Ca}$ mera alta per il ricorso al President's Rule.

Diversamente dalla prima variabile (il principio di rappresentanza nella Camera alta), la seconda variabile, relativa alle competenze legislative della Camera alta, non è quantificabile. Credo tuttavia che le evidenze di natura qualitativa appena presentate consentano egualmente, così come appare nella tab. 2, di collocare i cinque paesi considerati lungo il continuum demovincolante.

Quantum di attribuzione del policy making alle sotto-unità della federazione. La nostra terza variabile è una variabile complessa che comprende tre componenti strettamente collegate tra loro, ma analiticamente distinte. La prima componente riguarda il numero di materie legislative incluse nella Costituzione e che richiedono maggioranze legislative eccezionali. La seconda riguarda la distribuzione dei poteri tra le sotto-unità e il livello centrale. La terza componente, infine, tiene conto di dove risiede il potere legislativo - se a livello centrale o delle sotto-unità - quando la Costituzione non si pronuncia.

Se la Costituzione è relativamente parsimoniosa o se si limita ad indicare i principi generali, la maggior parte delle leggi può essere approvata a maggioranza semplice. In base alla seconda variabile, la votazione può coinvolgere o meno la Camera alta.

20 Per la storia e l'evoluzione recente del President's Rule in India si vedano Marwah (1995) e Mozoomdar (1995). Intendo sviluppare più diffusamente questo punto quando scriverò i risultati del mio viaggio in India compiuto nell'aprile del 1997, in cui ho discusso dell'art. 356 con l'ex Presidente della Corte suprema e con l'ex Primo ministro di Kerala contro cui tale procedura era stata utilizzata. Entrambi ritengono che si debba fare un uso più parsimonioso di questa norma. 
TAB. 2. Prerogative costituzionali della Camera alta nei confronti della maggioranza di governo a livello centrale

\begin{tabular}{|c|c|c|c|c|}
\hline Vincolo minimo & & & & Vincolo massimo \\
\hline $\begin{array}{l}\text { (eccessivamente } \\
\text { asimmetrico) }\end{array}$ & (meno asimmetrico) & (asimmetrico) & (più simmetrico) & $\begin{array}{l}\text { (eccessivamente } \\
\text { simmetrico) }\end{array}$ \\
\hline $\begin{array}{l}\text { La Camera territoriale non } \\
\text { ha poteri costituzionali per } \\
\text { proteggere l'autonomia del- } \\
\text { le unità territoriali contro } \\
\text { un intervento del governo } \\
\text { centrale di } 60 \text { giorni. La } \\
\text { Camera alta ha la facoltà di } \\
\text { sottoporre a revisione o ri- } \\
\text { cusare il governo del Presi- } \\
\text { dente solo dopo } 60 \text { giorni. } \\
\text { Si tratta di una Camera di } \\
\text { revisione }\end{array}$ & $\begin{array}{l}\text { L'art. } 155 \text { della Costituzio- } \\
\text { ne preclude l'intervento del } \\
\text { governo centrale a meno } \\
\text { che esso non ottenga la } \\
\text { maggioranza assoluta dei } \\
\text { consensi nella Camera alta. } \\
\text { La Camera alta non parte- } \\
\text { cipa al voto di sfiducia co- } \\
\text { struttivo né alla legislazione } \\
\text { ordinaria. } \\
\text { Si tratta di una Camera di } \\
\text { revisione }\end{array}$ & $\begin{array}{l}\text { La Camera alta non parte- } \\
\text { cipa al voto di sfiducia co- } \\
\text { struttivo, che è di esclusiva } \\
\text { competenza della Camera } \\
\text { bassa. Può solo svolgere un } \\
\text { ruolo potenziale di veto in } \\
\text { circa metà dell'agenda legi- } \\
\text { slativa totale che riguarda } \\
\text { direttamente le relazioni } \\
\text { tra centro e periferie. Il po- } \\
\text { tere della Camera alta è in } \\
\text { qualche modo aumentato } \\
\text { negli ultimi vent'anni. I } \\
\text { conflitti tra le due Camere } \\
\text { sono in genere risolti in } \\
\text { modo relativamente con- } \\
\text { sensuale all'interno di com- } \\
\text { missioni chiuse dove la Ca- } \\
\text { mera bassa ha la maggio- } \\
\text { ranza dei } 2 / 3 \text {. }\end{array}$ & $\begin{array}{l}\text { Estesa capacità di bloccare } \\
\text { una maggioranza democra- } \\
\text { tica. Su tutta la legislazione } \\
\text { la Camera non rappresen- } \\
\text { tativa ha gli stessi diritti di } \\
\text { voto della Camera bassa, in } \\
\text { cui vige il principio «una } \\
\text { persona, un voto». Il Sena- } \\
\text { to ha la competenza esclu- } \\
\text { siva di confermare o rifiu- } \\
\text { tare tutte le principali no- } \\
\text { mine giudiziarie e ammini- } \\
\text { strative. Un presidente di } \\
\text { una commissione può a } \\
\text { volte costituire un gruppo } \\
\text { vincente composto da una } \\
\text { sola persona. Un gruppo } \\
\text { vincente di senatori che } \\
\text { rappresentano solo il } 15 \% \\
\text { dell'elettorato totale può } \\
\text { bloccare la legislazione or- } \\
\text { dinaria. }\end{array}$ & $\begin{array}{l}\text { Prerogative eccessive per il } \\
\text { funzionamento efficace e } \\
\text { legittimo del governo de- } \\
\text { mocratico. La Camera alta } \\
\text { estremamente dispropor- } \\
\text { zionale deve approvare tut- } \\
\text { ta la legislazione. Vi sono } \\
12 \text { aree in cui il Senato ha } \\
\text { prerogative legislative esclu- } \\
\text { sive. Un gruppo vincente } \\
\text { di senatori che rappresen- } \\
\text { tano il } 13 \% \text { dell'elettorato } \\
\text { totale puo bloccare la legi- } \\
\text { slazione ordinaria sostenu- } \\
\text { ta da senatori che rappre- } \\
\text { sentano l'87\% della popo- } \\
\text { lazione. }\end{array}$ \\
\hline
\end{tabular}


Tuttavia le tre componenti sopra menzionate possono interrelarsi in modo tale che senatori che rappresentano meno dell' $8 \%$ dell'elettorato possono avere un potere di veto su un ampio numero di questioni legislative e possono opporsi con successo alla maggioranza anche più volte. Non si tratta solo di una questione di teoria democratica, ma anche di prassi. Se una minoranza può usare il suo potere di veto per difendere lo status quo che i teorici della democrazia, i costituzionalisti, la pubblica opinione e la maggioranza della classe politica ritengono vada modificato, allora si è in presenza di un pericolo per la democrazia. Tale situazione potrebbe creare un clima di diffusa semi-lealtà verso la democrazia stessa e/o condurre ad un contesto che, con le parole di O'Donnell, potremmo definire di «democrazia delegativa» (delegative democracy), in cui i presidenti governano con misure eccezionali in nome dell'efficacia, ai danni però dell'intero processo democratico (O'Donnell 1994) ${ }^{21}$.

Questa variabile riguarda anche la quantità di policy making riservato, rispettivamente, al centro e alle sotto-unità. I sistemi federali democratici variano enormemente riguardo a questo aspetto. A questo proposito è necessario fare una importante distinzione. Un sistema politico può decentrare l'amministrazione e trasferire la maggior parte del bilancio alle sotto-unità in ambiti come la sanità, la messa in opera delle leggi e il welfare, ma riservare al centro il diritto di approvare la legislazione di base e l'esercizio di controlli autoritativi in quelle stesse aree. Oppure il sistema può attribuire alle sotto-unità, in quelle stesse aree, poteri legislativi e di imposizione fiscale esclusivi, indipendentemente dal fatto che contribuiscano all'uguaglianza dei cittadini e al benessere della federazione.

Anche in questo caso, rispetto a tutte e tre le componenti, il Brasile risulta essere il paese più demo-vincolante. Un deluso ex ministro della pianificazione, José Serra - autorevole economista e senatore votato con un consenso elettorale senza precedenti nella storia del Brasile - ha affermato a proposito della Costituzione del 1988: «Non si tratta di una vera Costituzione, ma di una legge sociale, politica ed economica» ${ }^{22}$. Il testo costituzionale si occupa infatti di un numero enorme di questioni (i pro-

21 Sul concetto di «semi-lealtà» e su come esso possa contribuire al crollo della democrazia si veda Linz (1978).

22 Intervista dell'autore a J. Serra del 2 dicembre 1996 (San Paolo); l'affermazione è stata ripetuta in un intervento pubblico tenutosi all'Università di Londra nel febbraio 1997. 
grammi pensionistici; il regime dei beni pubblici e dei beni di proprietà estera; le imposte speciali per lo sviluppo di progetti regionali; i criteri di ripartizione delle entrate erariali tra il centro, gli Stati e i comuni; e così via) fin nei dettagli ${ }^{23}$. Tali questioni trascendono dunque la portata della legislazione ordinaria. Per emendare una qualsiasi di queste materie è richiesto pertanto il voto favorevole del $60 \%$ di tutti i membri (assenti compresi), per due volte, in entrambe le Camere. In un paese grande come un continente, in cui persino nell'Assemblea costituente il tasso medio di assenze era superiore al $30 \%$, si tratta di un obiettivo proibitivo. A dire il vero, tale procedura di emendamento della Costituzione è più facile di quella prevista negli Stati Uniti, ma la Costituzione americana è assai più parsimoniosa.

Anche ipotizzando la presenza di tutti i parlamentari - il che implicherebbe una straordinaria azione di lobbying e di ricompense laterali per il governo - il minimo «gruppo vincente» per bloccare qualsiasi legge di rilevanza costituzionale è costituito da un numero di senatori che rappresentano l' $8 \%$ dell'elettorato totale ${ }^{24}$. In questa situazione entra in gioco il meccanismo definito da Carl Friedrich «legge delle reazioni previste». La situazione brasiliana rappresenta un chiaro esempio di ciò che Shepsle e Weingast chiamano «equilibrio strutturalmente indotto». Dal momento che tutti gli attori coinvolti sono consapevoli del potenziale di veto di una piccola minoranza, molte delle potenziali decisioni politiche vengono eliminate dall'agen$\mathrm{da}$ anche se sono sostenute da una maggioranza del congresso e dall'opinione pubblica ${ }^{25}$. Quando poi una questione non è can-

23 Questo si è verificato perché la Costituzione del 1988 è stata approvata dopo un lungo periodo di governo militare (1964-1985), durante il quale il potere degli stati è stato notevolmente limitato. Nella nuova atmosfera democratica dell'Assemblea costituente si è cominciato a pensare che più i diritti sarebbero stati garantiti esplicitamente ed il potere devoluto agli Stati e alle municipalità, più il Brasile sarebbe stato democratico. Inoltre, gli interessi del livello federale di governo non sono stati efficacemente rappresentati nell'Assemblea costituente perché il Brasile non ha avuto un Presidente eletto direttamente dal 1960. Invece i governatori, eletti direttamente a partire dal 1982, hanno avuto un grande potere morale e politico nell'Assemblea costituente e la stessa Costituzione decentrata, che ha trasferito una quantità significativa del prelievo fiscale del Brasile dal livello centrale agli stati e ai municipi, ha dato soddisfazione a molti dei loro interessi politici, finanziari e fiscali.

24 Sono arrivato a questo dato in base al seguente ragionamento: più del $40 \%$ dei voti necessari a bloccare la riforma costituzionale sono espressi da senatori che rappresentano solo l'8\% della popolazione brasiliana.

25 Questo punto è stato spesso enfatizzato durante le interviste che ho tenuto tra il novembre e il dicembre 1996 con numerosi esponenti del governo centrale, fra i quali il Ministro delle finanze, il Presidente e l'ex ministro della Pianificazione. 
cellata dall'agenda, i costi dell'approvazione di una legge di riforma in termini di log-rolling e pagamenti regionali straordinari sono spesso assai onerosi. Per un paese come il Brasile, che non più tardi del 1993 aveva un tasso d'inflazione annuo superiore al $2000 \%$, un tale modello di paralisi indotta strutturalmente e/o di azioni ad alti costi si traduce in un enorme vincolo alla capacità del governo di sviluppare una coerente pianificazione fiscale e di realizzare le riforme ritenute necessarie.

L'altra componente costituzionalizzata riguarda il potere degli Stati e delle municipalità del paese. Ventiquattro dei ventisette Stati brasiliani hanno una propria Banca centrale che spesso concede prestiti agli Stati stessi. L'unico organo federale che deve approvare i relativi titoli di Stato è la Camera territoriale, ossia il Senato. Fino al novembre 1996, gli Stati avevano anche il potere di tassare le esportazioni; il costo per ottenere dal Congresso federale l'abolizione di tale norma è stato stimato in mezzo punto percentuale del Pil brasiliano del 1997.

L'ultima componente dell'autorità delle sotto-unità riguarda il potere residuale di fare le leggi. In Brasile si presume che se la Costituzione non si pronuncia, il potere legislativo residuale spetta alle sotto-unità ${ }^{26}$. Nel caso brasiliano, l'interazione tra le prime tre variabili crea un potenziale di veto nei confronti della maggioranza assai elevato e ciò conferma la collocazione di tale paese all'estremo del continuum che stiamo analizzando.

Gli Stati Uniti condividono con il Brasile la presunzione che negli ambiti non espressamente esaminati dalla Costituzione la sovranità residuale risieda negli Stati. Tale presunzione è di per sé demo-vincolante. Il suo impatto politico è tuttavia, negli Stati Uniti, meno vincolante che non in Brasile, dal momento che la Costituzione americana è più parsimoniosa e che nessuno Stato ha una propria Banca d'emissione.

La Costituzione tedesca è molto meno demo-vincolante di quella americana. Al governo centrale sono espressamente attribuite ampie aree di potere legislativo. Inoltre, parte della legislazione è concorrente, ovvero condivisa dal centro e dalle sotto-unità. Ancora, la Costituzione è esplicita e pertanto riduce il potere delle Corti di fare leggi attraverso le poche parole dell'art. 31: «la legge federale ha la precedenza sulle leggi statali».

26 Per una pionieristica analisi sulla Costituzione del 1988 e sui poteri dei governatori si veda Abruçio (1996). 
Anche gli artt. 72-74 chiariscono le aree in cui la legge federale può prevalere, se la Camera bassa decide in tal senso. Una delle innovazioni nella prassi del federalismo tedesco è che mentre la maggioranza nella Camera bassa mantiene il potere costituzionale di approvare le leggi e di esercitare controllo, la percentuale di imposte incassate che viene effettivamente spesa dai Länder è maggiore di quella spesa dagli Stati degli Stati Uniti. La maggior parte dei programmi federali, inoltre, è direttamente amministrata dall'apparato burocratico dei Länder, mentre negli Stati Uniti molti programmi federali sono amministrati dalla burocrazia federale. Si può pertanto affermare che in Germania la Camera bassa detiene più potere legislativo che non negli Stati Uniti, ma decentra maggiori responsabilità amministrative. Il punto chiave è che un sistema politico può decentrare senza che la cittadinanza, a livello centrale, perda costituzionalmente il potere legislativo e di controllo.

L'aspetto più significativo del federalismo spagnolo riguar$\mathrm{da}$ la dimensione verticale. Il sistema spagnolo delle autonomie è un sistema di «federalismo asimmetrico» nel senso che alcune Comunità, come la Catalogna e i Paesi Baschi, hanno negoziato, attraverso gli Statuti di autonomia, maggiori prerogative. Tali prerogative sono inserite in Statuti di rilevanza costituzionale. Dal punto di vista della teoria dei giochi, il federalismo asimmetrico spagnolo ha dato luogo ad un gioco iterativo di contrattazione. Un leader politico nazionalista, ad esempio, potrebbe guidare un partito nazionalista che ha la sua base in una Comunità $i$ cui voti sono necessari per mettere insieme una maggioranza di governo al livello centrale. Questa situazione offrirebbe di fatto alla Comunità in questione il potere di contrattazione per rinegoziare $\mathrm{i}$ termini dello Statuto di autonomia. Finora questi giochi di contrattazione propri di un federalismo asimmetrico come quello spagnolo sono avvenuti all'interno della Costituzione.

Anche il federalismo russo è asimmetrico; tuttavia la maggior parte dei giochi iterativi di contrattazione rimangono al di fuori della Costituzione. I giochi di contrattazione della Russia federale, pertanto, mettono a rischio il consolidamento democratico, dal momento che le sotto-unità riformulano unilateralmente le loro Costituzioni in modi che spesso violano la Costituzione della federazione russa.

La Costituzione indiana mantiene il potere residuale al livello centrale ma, per molti aspetti, è una costituzione che favori- 
sce il federalismo plurinazionale indiano. Permette ad esempio alla maggioranza al centro e a gruppi di minoranze linguistiche nelle sotto-unità di lavorare insieme per creare con grande facilità, sotto l'autorità parlamentare, nuovi stati linguistici ${ }^{27}$. Questa capacità si è rivelata estremamente importante nel «mantenere unita» l'India in modo democratico. Proprio perché i membri della Assemblea costituente erano consapevoli che la questione più controversa dell'unità indiana era quella linguistica e che c'era il desiderio da parte di molti delegati di riorganizzare gli stati in base alle linee linguistiche, il linguaggio della Costituzione è stato estremamente demo-emancipante. Ai parlamenti futuri è stato assegnato il potere di ridefinire completamente i confini statali. Su questo punto l'art. 3 della Costituzione è stato categorico. Con una semplice maggioranza «il parlamento può per legge a) formare uno stato attraverso la separazione del territorio di qualsiasi stato o attraverso l'unione di più stati...; c) ridurre la superficie di ogni stato...; e) cambiare il nome di ogni stato». In un vero sistema federale del tipo «per diventare uniti» gli stati sovrani sarebbero ovviamente stati capaci di accordarsi con successo per una Costituzione più demovincolante in grado di proteggere $\mathrm{i}$ diritti degli stati. Il censimento del 1991 indica che l'India ha più di 3.000 lingue madri legalmente riconosciute. $\mathrm{Ci}$ sono 33 lingue differenti ognuna delle quali è parlata da almeno un milione di persone. Tra il 1956 e il 1966, in modo relativamente consensuale, sono stati ridefiniti i confini della maggior parte degli Stati indiani e, in seguito, un processo di creazione di nuovi stati tribali ha preso avvio nel Nord-est.

In termini politici è probabilmente giusto riconoscere che la sopravvivenza dell'India come democrazia plurinazionale e multiculturale più grande del mondo è stata facilitata dalla struttura costituzionale del sistema federale. La struttura demo-emancipante del federalismo indiano ha consentito alla maggioranza al

27 Per l'approccio multifattoriale che ha guidato quella che è diventata la più ampia ridefinizione al mondo dei confini linguistici, sociali, ambientali e politici, cfr. Government of India (1955). Per un'analisi della politica linguistica in India si veda Brass (1974). Per comprendere le tensioni secessionistiche sorte nelle aree Tamil del Sud prima della devoluzione linguistica per «mantenersi uniti» si veda Barnett (1976). Vi sono state anche mobilitazioni e proteste nel Sud di lingua non Hindi affinché nell'Unione indiana l'inglese venisse mantenuto come lingua ufficiale di comunicazione. Si veda al proposito Forrester (1965). Come conseguenza della politica del federalismo democratico, tanto l'inglese quanto l'bindi vennero mantenute come lingue ufficiali. 
TAB. 3. Quantum di attribuzione costituzionale del policy making alle sotto-unità della federazione

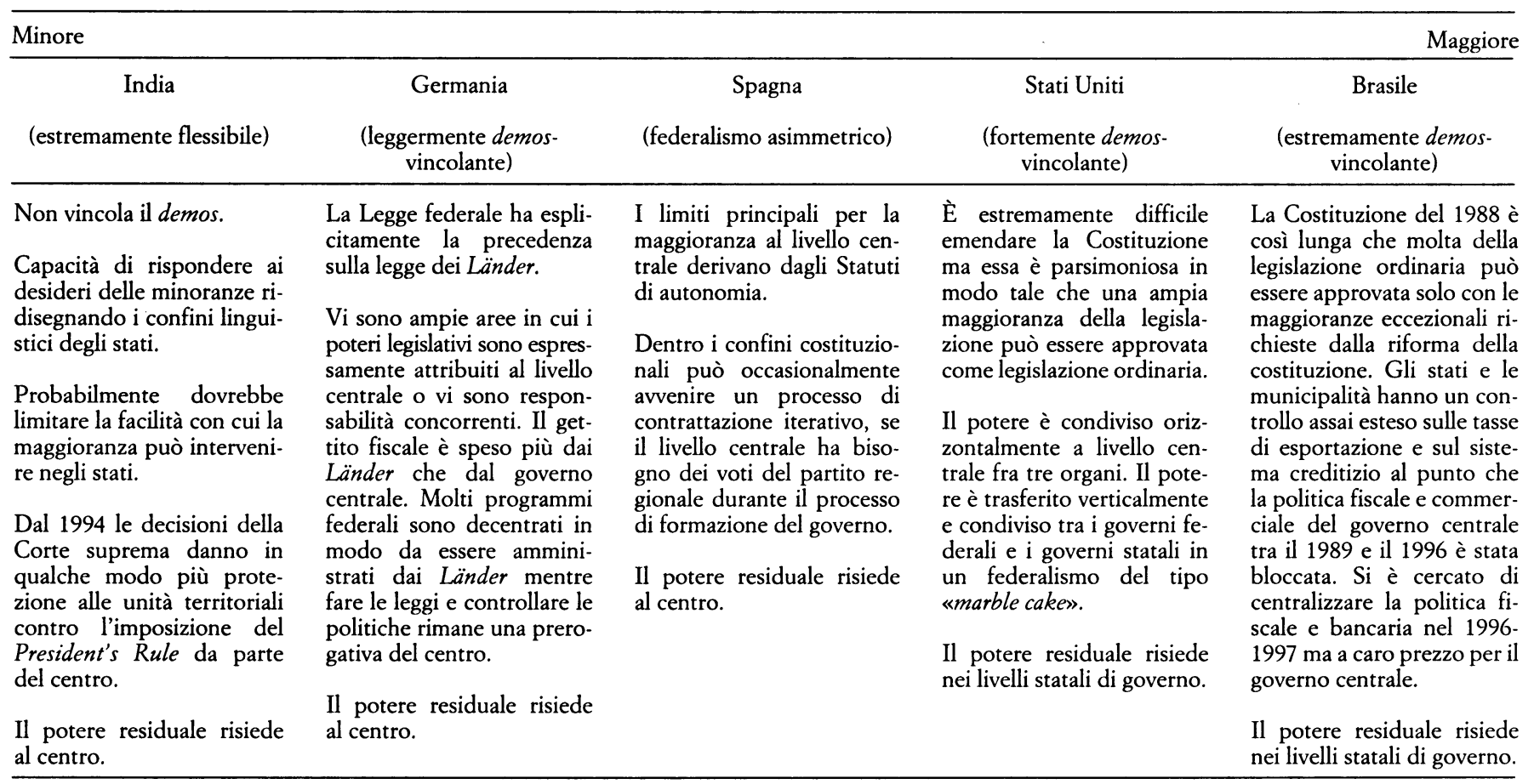


centro di rispondere alle richieste di una sempre maggiore autonomia linguistica e culturale delle minoranze degli Stati. Se l'India fosse stata uno stato unitario, né la maggioranza né le minoranze avrebbero avuto a loro disposizione questa flessibilità costituzionale. Quanto al continuum riguardante la terza variabile, esso è illustrato nella tabella 3.

Grado in cui il sistema di incentivi e l'orientamento del sistema partitico sono nazionalizzati. Un sistema federale per definizione ha un esecutivo e un legislativo eletti in ognuna delle unità costituenti. Questi esecutivi e legislativi necessariamente hanno funzioni di controllo sul bilancio e sulle leggi. Strutturalmente ed empiricamente, pertanto, il federalismo di per sé è una fonte di patronage, potere e prestigio per fronteggiare il potere e l'autorità dei partiti nazionali. Queste sfide sono presenti in modo sistematicamente inferiore nei sistemi unitari rispetto a quelli federali. Alcuni studiosi evincono da ciò che la relativa assenza di ideologia e di partiti fortemente disciplinati negli Stati Uniti dipenda fondamentalmente dal sistema federale (Truman 1955). Questo è vero per gli Stati Uniti ma non è vero per tutti i sistemi federali.

Per una serie di ragioni, alcuni sistemi partitici federali spesso producono a livello centrale una maggioranza (monopartitica o di coalizione). La coalizione può anche avere una disciplina nazionale e una forte infrastruttura organizzativa che produce incentivi per un sostegno disciplinato ai partiti nazionali. In questi casi il sistema partitico può agire come una forza centralizzante in grado di produrre una maggioranza sia in una federazione mono-nazionale come la Germania dal 1949, sia in una federazione multiculturale e multilinguistica come l'India tra il 1947 e il 1967.

Ci sono molti fattori di compensazione, tra cui l'ideologia, che possono contribuire ad una tale unità nazionale nonostante le intrinseche spinte alla frammentazione proprie di tutti i sistemi federali. L'assenza di elezioni primarie, un sistema proporzionale a lista bloccata, un sistema maggioritario in cui il partito nazionale seleziona i candidati nei collegi uninominali, un finanziamento ai candidati locali proveniente per lo più dal partito nazionale, la presenza di un sistema parlamentare sono fattori di questa natura. In tali casi potrà essere disponibile un insieme di incentivi strutturali e di scelta razionale che - nonostante il federalismo - produrranno partiti disciplinati e nazionali. Se, al 
contrario, si è in presenza di un sistema proporzionale a lista aperta, di collegi uninominali con elezioni primarie, se i candidati si finanziano la campagna elettorale in modo autonomo e indipendentemente dal partito nazionale e se il sistema è presidenziale, allora gli incentivi strutturali e di scelta razionale in grado di favorire partiti nazionali sono assai limitati. Gli Stati Uniti e ancora di più il Brasile sono sistemi di questo secondo tipo (Mainwaring, 1998) 28 .

Il Brasile e gli Stati Uniti, però, sono «nazioni-stato». La situazione è potenzialmente più complicata (e democraticamente più a rischio) se si tratta di stati plurinazionali e se non ci sono partiti nazionali disciplinati. In Russia, ad esempio, 103 dei 171 membri del Consiglio federale nel 1997 non appartenevano ad alcun partito (Barber 1997). Sia in Yugoslavia che in Unione Sovietica, entrambi paesi multinazionali, le prime elezioni competitive si sono tenute non a livello centrale bensì a livello provinciale. In entrambi i casi, l'assenza di partiti nazionali ha contribuito alla disintegrazione dello stato (Linz e Stepan 1992). Al contrario, per quanto riguarda la «nazione-stato» federale del Brasile, il fatto che, durante il processo di transizione, si siano svolte elezioni dirette per la scelta dei governatori nel 1982, ma non per la presidenza (almeno fino al 1989) ha contribuito alla natura vincolante del centro e del demos della Costituzione brasiliana del 1988 e al crescente decentramento delle risorse fiscali, ma non ha messo in pericolo l'unità territoriale del paese.

La Spagna è uno stato federale plurinazionale con forti partiti nazionali e alcuni partiti regionali piccoli ma rilevanti. Linz ed io abbiamo sostenuto altrove che il fatto che le prime elezioni libere dopo la morte di Franco siano state nazionali abbia contribuito alla creazione di forti partiti nazionali persino in Catalogna e nei Paesi Baschi (Linz e Stepan 1992). Tuttavia la Catalogna, comunità con il movimento autonomo sociologicamente più forte, è governata da una coalizione di partiti regionali e nazionali. Il partito regionale è forte anche nei Paesi $\mathrm{Ba}$ schi. Questa è una caratteristica fondamentale del sistema di federalismo asimmetrico della Spagna e contribuisce al gioco iterativo di contrattazione che si verifica quando nessun partito a livello nazionale ha la maggioranza.

28 Il Brasile si colloca vicino ai valori massimi riguardo ad un grande numero di indicatori riguardanti la frammentazione partitica e l'assenza di disciplina partitica. 
TAB. 4. La tendenza del sistema partitico a mitigare, in base al suo orientamento nazionale, le caratteristiche demo-vincolanti del federalismo

\begin{tabular}{|c|c|c|c|c|c|}
\hline Maggiore & & & & & Minore \\
\hline $\begin{array}{l}\text { Forti partiti nazionali } \\
\text { controllano quasi tutti } \\
\text { i seggi nella Camera } \\
\text { bassa e alta ed esercita- } \\
\text { no una rigida discipli- } \\
\text { na sui loro membri. } \\
\text { Tradizione ideologica } \\
\text { di fiducia e aiuto reci- } \\
\text { proco (Bundestreue). }\end{array}$ & $\begin{array}{l}\text { Il Partito del Congres- } \\
\text { so, nazionale, ha una } \\
\text { forte maggioranza a li- } \\
\text { vello centrale e con- } \\
\text { trolla la grande mag- } \\
\text { gioranza degli stati, in } \\
\text { cui svolge un ruolo im- } \\
\text { portante ma non coer- } \\
\text { citivo nelle nomine. }\end{array}$ & $\begin{array}{l}\text { I partiti nazionali con- } \\
\text { trollano la maggior } \\
\text { parte della Camera } \\
\text { bassa che è quella cru- } \\
\text { ciale per il processo le- } \\
\text { gislativo. Ma a causa } \\
\text { del federalismo pluri- } \\
\text { nazionale asimmetrico } \\
\text { vi sono forti partiti re- } \\
\text { gionali che sono in gra- } \\
\text { do di negoziare uno } \\
\text { status speciale quando } \\
\text { nessun partito ha la } \\
\text { maggioranza al livello } \\
\text { centrale. }\end{array}$ & $\begin{array}{l}\text { Il sistema delle prima- } \\
\text { rie e l'auto-finanzia- } \\
\text { mento rendono l'in- } \\
\text { fluenza locale e statale } \\
\text { più importante dell'in- } \\
\text { fluenza nazionale sia } \\
\text { nelle nomine che nelle } \\
\text { elezioni. } \\
\text { Polsby definisce quello } \\
\text { americano un sistema } \\
\text { «con } 100 \text { partiti statali } \\
\text { dietro due stendardi». } \\
\text { Il sistema di nomina del } \\
\text { presidente favorisce un } \\
\text { sistema bipartitico così } \\
\text { come la legislazione } \\
\text { elettorale statale. Nel } \\
\text { sistema legislativo fede- } \\
\text { rale il sistema bipartiti- } \\
\text { co è incoraggiato dalle } \\
\text { regole della Commis- } \\
\text { sione congressuale che } \\
\text { privilegiano i due parti- } \\
\text { ti principali. La disci- } \\
\text { plina partitica è cre- } \\
\text { sciuta nel partito De- } \\
\text { mocratico con la fine } \\
\text { dei Dixicrates negli anni } \\
\text { '60 e nel partito Repub- } \\
\text { blicano a causa del- } \\
\text { l'emergere dei neo-con- } \\
\text { servatori con Reagan. }\end{array}$ & $\begin{array}{l}\text { Nessun partito al livel- } \\
\text { lo centrale ha avuto la } \\
\text { maggioranza. } \\
\text { La maggioranza degli } \\
\text { Stati è governata da } \\
\text { partiti di fatto regiona- } \\
\text { li. } \\
\text { Tredici partiti, la mag- } \\
\text { gior parte dei quali re- } \\
\text { gionali ma non separa- } \\
\text { tisti, ha dato vita ad un } \\
\text { governo al livello cen- } \\
\text { trale nel 1996-1997. }\end{array}$ & $\begin{array}{l}\text { Le nomine sono decise } \\
\text { di fatto a livello statale } \\
\text { e municipale. } \\
\text { La maggior parte delle } \\
\text { campagne elettorali } \\
\text { sono auto-finanziate. } \\
\text { Punteggio estremamen- } \\
\text { te alto dell'indice di vo- } \\
\text { latilità partitica di Pe- } \\
\text { dersen ( } 50 \% \text { ). } \\
\text { Ampio numero di par- } \\
\text { titi. Indice di Laasko- } \\
\text { Taagepera intorno a } 8 \text {. } \\
\text { Quasi un terzo dei de- } \\
\text { putati eletti al Congres- } \\
\text { so nella legislatura } \\
\text { 1992-1996 hanno cam- } \\
\text { biato partito. } \\
\text { «Nazione-stato» per } \\
\text { cui non ci sono partiti } \\
\text { separatisti ma molte } \\
\text { delegazioni statali a li- } \\
\text { vello centrale hanno } \\
\text { forti agende di politica } \\
\text { regionale. }\end{array}$ \\
\hline
\end{tabular}


La Russia, come la Spagna, è un esempio di federalismo asimmetrico, ma differisce da quest'ultima in molti aspetti, alcuni dei quali molto importanti e rischiosi per la democrazia. La Spagna è un sistema parlamentare e dal 1977 ha contato sulla presenza di un partito quasi maggioritario, il cui leader è diventato Primo ministro e ha dato impulso alla disciplina nazionale del proprio partito. In Russia il sistema è semi-presidenziale dal 1991. Il mandato presidenziale deriva da una elezione diretta e pertanto è indipendente dal fatto che il Presidente sia sostenuto o no dalla maggioranza in Parlamento. In effetti, il Presidente Yeltsin (che non ha mai avuto una maggioranza alla Camera bassa) non ha fatto campagna per i partiti politici nell'ultima elezione e molti dei candidati per la Duma e per i governatorati si sono presentati come «indipendenti». Come in Spagna, il federalismo asimmetrico russo ha prodotto un gioco iterativo di contrattazione, ma con effetti maggiormente frenanti rispetto al processo di consolidamento democratico. Molte sotto-unità russe hanno cercato unilateralmente di riformulare la propria costituzione, spesso violando la costituzione federale. La tabella 4 presenta l'effetto più o meno mitigante del sistema partitico rispetto alle caratteristiche demo-vincolanti dei rispettivi sistemi federali.

\section{Osservazioni conclusive}

Le istituzioni federali sono rilevanti sul piano delle politiche pubbliche in tutti i livelli del continuum demo-vincolante. All'estremo superiore, $i$ senatori brasiliani che rappresentano appena l' $8 \%$ dell'elettorato hanno più seggi dei senatori che rappresentano oltre il $90 \%$ dell'elettorato. Il federalismo asimmetrico «per mantenersi uniti» della Spagna produce un gioco iterativo di contrattazione ma ha anche contribuito a generare quello che Linz ed io abbiamo altrove descritto come un sistema di identità multiple ma complementari. Nel 1991, in Catalogna, il $73 \%$ della popolazione era orgogliosa di essere spagnola, l' $82 \%$ di essere catalana e 1' $83 \%$ era favorevole a procedere verso formule federaliste all'interno della Comunità Europea (Linz e Stepan 1996, 102).

Persino all'estremo inferiore del continuum demo-vincolante il federalismo ha un impatto sulle politiche. Un sistema unitario non avrebbe potuto realizzare tanti e così rapidi aggiustamenti 
dei confini linguistici come è accaduto in India. Un sistema unitario, inoltre, non avrebbe probabilmente avuto a sua disposizione il vasto repertorio di politiche federali che permettono ad uno stato multiculturale, multilinguistico, multireligioso di un miliardo di persone come l'India di «mantenersi unito». In India, l'uso di strumenti federalizzanti creativi sembra, al tempo stesso, in grado di rispondere alle diversità e di ridurre in quasi tutti gli Stati le tendenze secessioniste a piccole minoranze. Il decentramento del potere su base democratica e federale e la concessione di diritti a gruppi specifici non ha creato un piano inclinato verso la secessione, né la violazione dei diritti individuali, come i teorici dei diritti liberali temevano ${ }^{29}$. Una volta che al popolo Tamil è stato dato uno stato, che l'inglese è rimasto la lingua ufficiale dell'Unione e che un partito regionale ha ottenuto il controllo del legislativo statale, la questione della secessione dei Tamil ha cessato di essere rilevante ${ }^{30}$. Le elezioni democratiche e il decentramento in Punjab alla fine degli anni '90 hanno reso una non-questione anche quella del violento movimento secessionista Sikh. L'India sembra più decentrata $\mathrm{e}$ più «nazione-Stato» con una cultura civilizzata alla fine degli anni '90 di quanto non lo sia mai stata prima. Identità multiple e complementari, che non erano la norma per alcuni importanti leader politici di lingua Tamil in India negli anni ' 50 o per un numero consistente di Sikh nel Punjab negli anni '80, sono ora la norma in entrambe le zone ${ }^{31}$. Questi processi dovrebbero essere attentamente valutati nello sviluppare una nuova agenda di ricerca sul federalismo democratico.

\section{[Traduzione di Franca Maino]}

29 Per un primo, pioneristico trattamento della questione dei diritti collettivi versus i diritti individuali, si veda Kymlicka (1995). Per una forte argomentazione sul fatto che i diritti di gruppo sono spesso una precondizione dei diritti individuali, si veda Raz (1986). Per una acuta discussione politica e filosofica di tali questioni in India si veda Bhargava (1994).

$30 \mathrm{Al}$ contrario, se lo stato unitario ma plurinazionale dello Sri Lanka dopo l'indipendenza avesse dato vita ad una federazione per «mantenersi uniti», con uno stato di lingua Tamil nel nord del paese, l'attuale ingestibile situazione di violenza etnica e collasso democratico probabilmente non sarebbe tale. Per un'analisi della costruzione politica delle identità polarizzate in Sri Lanka si veda Bose (1995).

31 Utili dati sulle lealtà e le identità a sostegno della democrazia in India sono contenute in una survey di prossima pubblicazione a cura di Singh e Yadev, del Center for the Study of Developing societies (Delhi). I risultati preliminari sono contenuti in India Today (1996). Si veda anche Kumar (1997) e Singh (1997). 
Riferimenti bibliografici

Abruçio, F.L. e D. Samuels (1997), The New Politics of the Governors: Subnational Politics and the Brazilian Transition to Democracy, relazione presentata al XVII Congresso dell'International Political Science Association, Seoul, Korea, 17-22 Agosto.

Agronoff, R. (1994), Asymmetrical and Symmetrical Federalism in Spain: An Examination of Intergovernmental Policy, in B. de Villiers (a cura di), Evaluating Federal Systems, London, Nijhoff Publishers, pp. 61-89.

Anderson, B. (1983), Imagined Communities: Reflections on the Origin and Spread of Nationalism, London, New Left Books.

Barber, J. (1997), Opposition in Russia, in «Government and Opposition», XXXII, 4, pp. 598-613.

Barnett, M.R. (1976), The Politics of Cultural Nationalism in South India, Princeton, Princeton University Press.

Bhargava, R. (1994), Secularism, Democracy and Rights, in M. Arslan e J. Rayan (a cura di), Communalism in India, Dehli, Manohan, pp. 61-73.

Bhattacharya, M. (1992), The Mind of the Founding Fathers, in N. Mukarji e B. Arora (a cura di), Federalism in India: Origins and Development, New Delhi, Vikas, pp. 81-102.

Blaustein, A.P. e G.H. Flanz (1991), Constitutions of the Countries of the World, Dobbs Ferry (NY), Oceana Publications.

Bose, S. (1995), State Crises and Nationalities Conflict in Sri Lanka and Yugoslavia, in "Comparative Political Studies», XXVIII, pp. 87. 116.

Brass, P.R. (1974), Language, Religion and Politics in North India, Cambridge, Cambridge University Press.

Copland, I. (1997), The Princes of India in the Endgame of Empire, 1917-1947, Cambridge, Cambridge University Press.

Dahl, R.A. (1986), Federalism and the Democratic Processes, in R.A. Dahl (a cura di), Democracy, Liberty and Equality, Oslo, Norwegian University Press, pp. 114-126.

- (1989), Democracy and its Critics, New Haven, Yale University Press, trad. it. La democrazia e i suoi critici, Roma, Editori Riuniti, 1990.

Elazar, D.J. (1987), Exploring Federalism, Tuscolosa, University of Alabama Press.

Elazar, D.J. et al. (1994), Federal System of the World: A Handbook of Federal, Confederal and Autonomy Arrangements, Harlow, Longman Group.

Elster, J. e R. Slagstad (a cura di) (1988), Constitutions and Democracy, Cambridge, Cambridge University Press.

Europa World Year Book 1990 (1990), Austria, The Constitution, London, Europa Publications. 
Europa World Year Book 1995 (1995), Germany, vol. 1, London, Europa Publications.

Figueiredo, A.C. e F. Limongi (1997), Medidas Provisorias: Abdicação ou Delegação?, in «Novos Estudos», XLVII, pp. 127-154.

Finer, H. (1956), Governments of Greater European Powers: A Comparative Study of the Governments and Political Culture of Great Britain, France, Germany and the Soviet Union, London, Methuen.

Finer, S.E., V. Bogdanor e B. Rudden (1995), Comparing Constitutions, Oxford, Clarendon Press.

Forrester, D.B. (1966), The Madras Anti-Hindi Agitation, 1965, in «Pacific Affairs», XXXIX, pp. 19-36.

Gellner, E. (1983), Nations and Nationalism, Oxford, Blackwell.

Goodin, R.E. (1996), Institutionalizing the Public Interest: The Defence of Deadlock and Beyond, in «American Political Science Review», XC, pp. 331-343.

Goodin, R.E. e H.D. Klingemann (a cura di) (1996), The New Handbook of Political Science, Oxford, Oxford University Press.

Government of India, Home Department (1955), Report of the States Reorganization Commission, New Dehli, Government of India Press.

Hooghe, L. (1993), Belgium: From Regionalism to Federalism, in «Regional Politics and Policy», 3, pp. 44-69.

India Constituent Assembly (1951), Constituent Assembly Debates, New Delhi, Vol. II.

Kumar, S. (1997), Punjab: A Vote for Change, in «Politics in India», I, 10.

Kymlicka, W. (1995), Multicultural Citizenship: A Liberal Theory of Minority-Rights, Oxford, Clarendon Press.

Lijphart, A. (1984), Democracies: Patterns of Majoritarian and Consensus Government in Twenty-one Countries, New Haven, Yale University Press, trad. it. Le democrazie contemporanee, Bologna, Il Mulino, 1988.

Linz, J.J. (1978), The Breakdown od Democratic Regimes: Crisis, Breakdown and Requilibration, Baltimora, Johns Hopkins University Press.

- (1989), Spanish Democracy and the Estado de las Autonomias, in R.A. Goldwin, A. Kaufman e W.A. Schambra (a cura di), Forging Unity out of Diversity: The Approach of Eight Nations, Washington DC, American Institute for Public Policy Research, pp. 260326.

- (1997), Democracy, Multinationalism and Federalism, relazione presentata al XVII Congresso dell'International Political Science Association, Seoul, Korea, 17-22 Agosto.

Linz, J.J. e A. Stepan (1992), Political Identities and Electoral Sequences: Spain, Soviet Union and Yugoslavia, in «Daedalus», CXXI, 2, pp. 510-522. 
- (1996), Problems of Democratic Transition and Consolidation: Southern Europe, South America and Post-Communist Europe, Baltimore, Johns Hopkins University Press.

Mainwaring, S. (1998), The Party System and Democratization in Brazil, Stanford, Stanford University Press, in corso di stampa.

Mainwaring, S. e D. Samuels (1997), Bringing the States Back In: Federalism and Democracy in Contemporary Brazil, relazione presentata alla Conferenza Democracy, Nationalism and Federalism, Oxford, 5-8 Giugno.

Marwah, V. (1995), Use and Abuse of Emergency Powers: The Indian Experience, in B. Arora e D.V. Verney (a cura di), Multiple Identities in a Single State: Indian Federalism in Comparative Perspective, New Dehli, Konark Publishers, pp. 136-159.

Menon, V.P. (1956), Integration of the Indian States, Madras, Orient Longman.

Miller, A.H., C. Tien, e A.A. Peebler, (1996), The American Political Science Review Hall of Fame: Assessments and Implications for an Evolving Discipline, in «Political Science \& Politics», XXIII, 1, pp. 73-83.

Mozoomdar, A. (1995), The Supreme Court and President's Rule, in B. Arora e D.V. Verney (a cura di), Multiple Identities in a Single State: Indian Federalism in Comparative Perspective, New Dehli, Konark Publishers, pp. 160-168.

O'Donnell, G. (1994), Delegative Democracy, in «Journal of Democracy», V, 1, pp. 55-69.

O'Donnell, G., P.C. Schmitter e L. Whitehead (a cura di) (1986), Transitions from Authoritarian Rule: Prospects for Democracy, Baltimora, Johns Hopkins University Press.

Ordershook, P.C. (1997), Russia's Party System: Is Russian Federalism Viable?, in «Post-Soviet Affairs», 12, pp. 195-217.

Ordershook, P.C. e O. Svetsova (1997), Federalism and Constitutional Design, in «Journal of Democracy», VIII, 1, pp. 27-42.

Przeworski, A. (1986), Some Problems in the Study of the Transition to Democracy, in G. O'Donnell, P.C. Schmitter e L. Whitehead (a cura di), Transitions from Authoritarian Rule: Prospects for Democracy, Baltimora, Johns Hopkins University Press, pp. 47-63.

Raz, J. (1986), The Morality of Freedom, Oxford, Clarendon Press.

Riker, W.H. (1964), Federalism: Origin, Operation, Significance, Boston, Little Brown.

- (1969), Six Books in Search of a Subject or Does Federalism Exist and Does it Matter?, in «Comparative Politics», II, 1, pp. 135-146.

- (1975), Federalism, in F. Greenstein e N.W. Polsby (a cura di), Handbook of Political Science, vol. 5, Governmental Institutions and Processes, Reading (Mass.), Addison-Wesley, pp. 93-172.

- (1980), Implications for the Disequilibrium of Majority Rule for the Study of Institutions, in «American Political Science Review», LXXIV, pp. 432-447. 
- (1982), Liberalism against Populism: A Confrontation between the Theory of Democracy and the Theory of Social Choice, San Francisco, Freeman.

- (1987a), The Development of American Federalism, Boston, Kluwer Academic Publishers.

- (1987b), The Invention of Centralized Federalism, in W.H. Riker, The Development of American Federalism, Boston, Kluwer Academic Publishers.

- (1996), European Federalism: The Lessons of Past Experience, in J.J. Hesse e V. Wright (a cura di), Federalizing Europe? The Costs, Benefits and Preconditions of Federal Political Systems, Oxford, Oxford University Press.

Senelle, R. (1996), The Reform of the Belgian State, in J.J. Hesse e V. Wright (a cura di), Federalizing Europe? The Costs, Benefits and Preconditions of Federal Political Systems, Oxford, Oxford University Press, pp. 266-324.

Singh, R. (1997), Politics in Punjab: Restoration of Accomodation Model, in «Politics in India», I, 10.

Shepsle, K.A. e B.R. Weingast (1981), Structure Induced Equilibrium and Legislative Choice, in «Public Choice», XXXVII, pp. 503-519.

- (1987), The Institutional Foundations of Committee Power, in «American Political Science Review», LXXXI, pp. 85-104.

Solnick, S.L. (1995), Federal Bargaining in Russia, in «East European Constitutional Review», IV, 4.

Stepan, A. (1997), Modern Multi-national Democracies: Trascending a Gellnerian Oxymoron, in J.A. Hall (a cura di), Ernest Gellner and the Theory of Nationalism, Cambridge, Cambridge University Press.

Suberu, R.T. (1997), The Paradoxes and Pathologies of Nigerian Federalism, relazione presentata alla Conferenza Democracy, Nationalism and Federalism, Oxford, 5-8 giugno.

Swenden, W. (1997), Belgium: A Federal State in Search of a Nation, relazione presentata alla Conferenza Democracy, Nationalism and Federalism, Oxford, 5-8 Giugno.

Swift, E.K. (1996), The Making of an American Senate: Reconstitutive Change in Congress, 1787-1841, Ann Arbor, University of Michigan Press.

Truman, D. (1955), Federalism and the Party System, in A.W. Macmahon (a cura di), Federalism: Mature and Emergent, Garden City, Doubleday, pp. 115-136.

Villiers, de B. (1995), Bundestreue: The Soul of an Intergovernmental Partnership: Comparative Analysis of the Principles Underlying Bundestreue in the Federal Republic of Germany, Switzerland and Belgium, Konrad-Adenauer Stiftung Occasional Papers RSA, marzo.

Wheare, K.C. (1946), Federal Government, Oxford, Oxford University Press.

Whitacker's Almanack (1997), London, Whitacker's. 